\title{
Two-scale $\Gamma$-convergence of integral functionals and its application to homogenisation of nonlinear high-contrast periodic composites
}

\begin{abstract}
An analytical framework is developed for passing to the homogenisation limit in (not necessarily convex) variational problems for composites whose material properties oscillate with a small period $\varepsilon$ and that exhibit high contrast of order $\varepsilon^{-1}$ between the constitutive, "stress-strain", response on different parts of the period cell. The approach of this article is based on the concept of "two-scale $\Gamma$-convergence", which is a kind of "hybrid" of the classical $\Gamma$-convergence (De Giorgi, E., Franzoni, T.: Atti Accad. Naz. Lincei Rend. Cl. Sci. Fis. Mat. Natur. (8) 58, 842-850, 1975) and the more recent two-scale convergence (Nguetseng, G.: SIAM J. Math. Anal. 20, 608-623, 1989). The present study focuses on a basic high-contrast model, where "soft" inclusions are embedded in a "stiff" matrix. It is shown that the standard $\Gamma$-convergence in the $L^{p}$-space fails to yield the correct limit problem as $\varepsilon \rightarrow 0$, due to the underlying lack of $L^{p}$-compactness for minimising sequences. Using an appropriate two-scale compactness statement as an alternative starting point, the two-scale $\Gamma$-limit of the original family of functionals is determined, via a combination of techniques from classical homogenisation, the theory of quasiconvex functions and multiscale analysis. Then related result can be thought of as a "non-classical" two-scale extension of the well-known theorem by S. Müller (Arch. Rational Mech. Anal. 99, 189-212, 1987).
\end{abstract}

Keywords homogenisation · calculus of variations $\cdot$ high-contrast composites · $\Gamma$-convergence $\cdot$ two-scale convergence

\author{
M. Cherdantsev \\ Cardiff University \\ Tel.: +44-2920-875549 \\ Fax: +44-2920-874199 \\ E-mail: CherdantsevM@cf.ac.uk \\ K. D. Cherednichenko \\ Cardiff University \\ Tel.: +44-2920-875540 \\ Fax: +44-2920-874199 \\ E-mail: CherednichenkoKD@cf.ac.uk
}




\section{Introduction}

Recent years have seen a number of advances in the development of various analytical techniques aimed at tackling multi-scale problems where the "classical" homogenisation theorems fail to hold. One prominent example of a problem of this sort is the linear periodic second-order elliptic partial differential equation (PDE) with high-contrast in the coefficients, which was considered by Zhikov [37], see also [6]. Suppose that the period $\varepsilon$ and the "contrast" $\delta^{-1}$ are scaled so that $\delta \sim \varepsilon^{2}$ as $\varepsilon \rightarrow 0$. A remarkable feature of the behaviour of the families of equations of this kind is that the two-scale character of the solution persists "in the very limit" as $\varepsilon \rightarrow 0$. Roughly speaking, in the classical two-scale expansion (see e.g. [4], [3])

$$
u_{\varepsilon} \sim u_{0}^{\delta}(x)+\varepsilon u_{1}^{\delta}(x, x / \varepsilon)+\varepsilon^{2} u_{2}^{\delta}(x, x / \varepsilon)+\ldots,
$$

where for simplicity we disregard the effects near the boundary ${ }^{1}$, all the terms become of the same order as $\varepsilon \rightarrow 0$ (and hence $\delta \rightarrow 0$ as well). This leads to the suggestion that in the expansion (1) $u_{0}$ can no longer be assumed independent of the "fast variable" $x / \varepsilon$ and must be expected to capture the leading-order oscillations on the scale of the small period of size $\varepsilon$.

The presence of the small-scale resonances in a system of this type can be quantified by proving that the spectrum of the associated operator converges in the sense of Hausdorff to the spectrum of a coupled "two-scale" limiting problem with respect to both the "slow" and "fast" variables. Further, the spectrum of this limiting problem is shown to have an infinite set of "band gaps", or intervals of frequencies at which wave propagation through the material sample is not allowed. This fact, which at a non-rigorous level had for some years been known to physicists, has caused an explosion of interest in the applied mathematics community towards "non-classical" materials, or "metamaterials", and continues to be actively explored, both theoretically and experimentally. Among popular applications of the distinctive properties of such "metamaterials" is the design of photonic (electromagnetic) and phononic (elastic) band-gap devices, which are capable of transmitting the corresponding kind of energy at large distances with significantly reduced losses compared to the usual transmission devices. Another recent trend is to exploit such materials for purposes of "invisibility", or "cloaking", of nearby objects, see [33], [22], [28].

From the mathematical perspective, the majority of the above developments has been set in the context of linear PDEs, which is traditionally the first class of equations to be explored. In many physics applications (such as the electromagnetics) the linear theory suffices, in some others (such as the high-frequency acoustics) it offers a good approximation to the behaviour of realistic objects. In the present article, however, we aim at the applied contexts that are inherently nonlinear, such as that of finite elasticity. It is well known that not only the linearised equations of elasticity but also any "elastic" models emerging from variational integrals with a convex stored-energy function fail to satisfy the principle of material frame indifference and hence have to be discarded for purposes of rigorous analysis.

\footnotetext{
${ }^{1}$ One way of making rigorous analysis possible in this setting is to consider a problem on a fixed-size torus, or "supercell" as numerical analysts would call it
} 
Most of the methods of classical homogenisation - two-scale asymptotic expansions [4], [3], Tartar's "energy method" [31], [19], the method of two-scale convergence ([1], [26]), $\Gamma$-convergence ([18], [8], [19]) etc.) - have been extended to treat a large class of nonlinear problems satisfying a number of technical constitutive assumptions, which have normally included some sort of "nondegeneracy" condition and have thus led to "classical" homogenised limits, i.e. those where the fast, "micro-scale", variable (represented by $x / \varepsilon$ in (1) above) is not explicitly present. The lack of a coupling effect between the macroscopic and microscopic length-scales in the limit as $\varepsilon \rightarrow 0$ seems to be inextricably linked to the fact that the kind of compactness argument that underpins the mathematical analysis in each of these contexts involves only functions of the macroscopic variable $x$. For instance, in the context of the two-scale convergence (see i.e. [1], [37]) it can be shown that if an $L^{p}$-bounded sequence $u_{\varepsilon}=u_{\varepsilon}(x)$ weakly two-scale converges to a function $u(x, y)$ of the slow $x$ and the fast $y$ variables and their gradients $\nabla u_{\varepsilon}$ are also $L^{p}$-bounded then the limit function $u$ is in fact independent of $y$ and the two-scale limit (after passing to a subsequence if necessary) of the gradients $\nabla u_{\varepsilon}$ differs from $\nabla u$ by the $y$-gradient of some two-scale function. Similarly, in the context of the classical $\Gamma$-convergence one usually deals with $W^{1, p}$-bounded sequences (more generally, $W^{k, p}$-bounded $(k \geq 1)$ if one thinks of a higher-order problem) of "almost minimisers", which are compactly embedded into $L^{p}$.

It is possible, however, to envisage practically motivated nonlinear settings where the stored-energy function is no longer non-degenerate, meaning that displacement fields whose gradient is "large" on a significant part of the material are compatible with the requirement that the total energy be finite, and can hence be viable candidates as "almost minimisers". The context of homogenisation seems to be a perfect environment for exploring such "nonlinear metamaterials": on the one hand, it is a promoted view at the moment that realistic stored-energy functions (and in general, any models) of continuous media are those that are obtained by some sort of averaging (or "homogenisation", "coarse graining") procedure from a model at a finer length-scale. On the other hand, the idea of introducing into the equations a high-contrast that is appropriately scaled with the microscopic size, has been a successful one in a number of physically relevant contexts mentioned above. In order to make the two-scale homogenisation work, one then first of all clearly needs a kind of "two-scale compactness" principle such as that employed in linear high-contrast problems. This is typically based on the observation that if an $L^{p}$-bounded sequence of functions $u_{\varepsilon}$ weakly two-scale converges to a function $u(x, y)$ (cf. the above "classical" compactness example), and the sequence of "non-classical" scaled gradients $\varepsilon \nabla u_{\varepsilon}$ is $L^{p}$-bounded, then the function $u(x, y)$ is in fact weakly differentiable in $y$ and, up to selecting a subsequence, the scaled gradients $\varepsilon \nabla u_{\varepsilon}$ two-scale converge to the $y$-gradient of $u$. The subsequent passage to the limit can then be performed in the weak formulation of the problem using e.g. the definition of the two-scale convergence, see for example a recent work [41] treating monotonic nonlinear high-contrast problems (i.e. problems whose stored-energy function has monotonic gradient). This procedure of passage to the limit, however, is bound to have an ad hoc character in general, since in most genuinely nonlinear contexts different weakly two-scale convergent subsequences will have different limits that $a$ priori do not satisfy the same limiting equation. One of the key difficulties in the related analysis is to demonstrate that the weak 
two-scale limit of the "fluxes" (i.e. the values of the gradient of the stored-energy function on the solution gradient) is the flux of the weak two-scale limit of the original sequences, which in the classical context is a key element of the compensated compactness principle of Murat and Tartar ([25], [31]), see also [40], [38], [11] for recent discussions of the related issues in various PDE contexts.

Following this unoptimistic observation, one is led to review the averaging tools mentioned above and notice that the abstract $\Gamma$-convergence approach, being an "ansatz-free" method, does not suffer from the lack of a compensated compactness argument. In its standard form, see [8], it does require the usual $L^{p}$ compactness principle but it seems to be extendible, however, to situations where a kind of non-classical "two-scale compactness" holds instead, as outlined above. It is the aim of the present work to provide such an extension. More precisely, consider a bounded domain (i.e. a connected open set) $\Omega \subset \mathbb{R}^{n}, n \geq 2$, the "unit cell" $Y:=[0,1)^{n}$, and a $Y$-periodic domain $E_{1} \subset \mathbb{R}^{n}$. Let $\varepsilon>0$, denote by $E_{1}^{\varepsilon}=\varepsilon E_{1}$ the " $\varepsilon$-contraction" of $E_{1}$, and set $E_{0}:=\mathbb{R} \backslash E_{1}, E_{0}^{\varepsilon}=\varepsilon E_{0}$ (see the next section for the precise definition of these sets). We study "high-contrast" variational integrals of the form

$$
I^{\varepsilon}(u):=\int_{\Omega}\left(W_{1}(\nabla u(x)) \chi_{E_{1}^{\varepsilon}}(x)+W_{0}(\varepsilon \nabla u(x)) \chi_{E_{0}^{\varepsilon}}(x)-f(x) \cdot u(x)\right) \mathrm{d} x,
$$

defined on weakly differentiable vector functions $u$ from the space $\left[L^{p}(\Omega)\right]^{m}, m \geq$ 2 . Here the functions $W_{1}, W_{0}$ represent the constitutive response of the material in question and $f \in\left[L^{q}(\Omega)\right]^{m}$ is the density of the body force, $p^{-1}+q^{-1}=1, f \cdot u$ is understood as the usual scalar product of vectors $f$ and $u$. We shall show that under some technical growth and continuity assumptions on the stored-energy functions $W_{i}(e), i=0,1$, the functionals $I^{\varepsilon}$ converge as $\varepsilon \rightarrow 0$ in an appropriately defined sense of "strong two-scale $\Gamma$-convergence" to the functional (cf. (46))

$$
I^{\text {hom }}(u)=\int_{\Omega}\left(W_{1}^{\mathrm{hom}}(\nabla u(x, y))+\int_{E_{0}} Q W_{0}\left(\nabla_{y} u(x, y)\right) \mathrm{d} y-f(x) \cdot u(x, y)\right) \mathrm{d} x,
$$

defined on a certain "two-scale" space of functions $u(x, y)$. Here $W_{1}^{\text {hom }}$ is the classical homogenised stored-energy function (see $e . g$. [19]) for the "perforated" material with the stored-energy function $W_{1}$ occupying the domains $\Omega \cap E_{1}^{\mathcal{E}}$ and $Q W_{0}$ is the quasiconvex envelope (see e.g. [17]) of the function $W_{0}$. This is the main result of the paper, which we formulate in Theorems 24 and 26.

Remark 1 The functionals (2) can be considered in conjunction with various boundary conditions on the function $u$. In what follows, we carry out our analysis for the case of "Neumann", or "traction-free" equilibrium configurations, where no boundary conditions on $\partial \Omega$ are additionally specified in (2), thus implying the widest possible underlying function space. This kind of setting is often referred to as the "analysis of arbitrary sequences". It may be pointed out however, that most of other common boundary conditions are also accounted for via a standard procedure (see e.g. [8]).

Further details of the problem we investigate are given in Section 2 of the present work. Before proceeding to the two-scale analysis of the functionals (2), in Section 3 we recall the standard definitions of $\Gamma$-convergence for sequences of 
functionals defined on $L^{p}(\Omega)$ and two-scale convergence of function sequences in $L^{p}(\Omega)$. We also provide a version of the "continuous extension lemma in Sobolev spaces on perforated domains, which we use in the subsequent analysis. In Section 4 we demonstrate via a simple example that the usual $\Gamma$-convergence does not suffice to obtain a proper variational limit of the functionals (2), and in Section 5 we introduce the notion of two-scale $\Gamma$-convergence, which later on is shown to yield the correct (two-scale) limit functional. Sections 6, 7, 8 contain the main technical component of the work, where we prove a statement (Theorem 24) regarding the two-scale $\Gamma$-convergence of the sequence (2) as $\varepsilon \rightarrow 0$ to a functional $I^{\text {hom }}$. In Sections 9 and 10 we prove the existence of a minimiser for $I^{\text {hom }}$ and the convergence of the infima of the functionals (2) to the minimum of $I^{\mathrm{hom}}$. Finally, Section 11 contains a discussion of the relationship between our results and other topics in applied analysis and of future extensions of our approach.

In conclusion to this introductory section we ought to point out that the technique we present here can be compared against that of the work [9]. The latter considers sequences of high-contrast functionals that are different from (2) in the way the contrast enters the stored-energy function. This difference between the two modelling approaches leads in the case of [9] to somewhat more restricted homogenisation theorems involving limit functionals with respect to the slow variable $x$ only, compared to our results, which provide a "full" two-scale limit (3).

\section{Problem setup}

We make the following assumptions regarding the functionals (2). The storedenergy functions $W_{1}$ and $W_{0}^{\varepsilon}$ are continuous and satisfy standard growth conditions $^{2}$ :

$$
\begin{aligned}
& \alpha\left(-c+|e|^{p}\right) \leq W_{1}(e) \leq \beta\left(1+|e|^{p}\right), \\
& \alpha\left(-c+|e|^{p}\right) \leq W_{0}^{\varepsilon}(e) \leq \beta\left(1+|e|^{p}\right) .
\end{aligned}
$$

We assume throughout the paper that $\beta \geq \alpha>0, c>0, p>1$. (More generally, we will henceforth say that $W(e)$ satisfies the standard growth conditions if $\alpha(-c+$ $\left.|e|^{p}\right) \leq W(e) \leq \beta\left(1+|e|^{p}\right)$, where the exponent $p$ is the same as in (4).) Functions $W_{0}^{\varepsilon}$ are assumed to converge pointwise to some continuous $W_{0}$ as $\varepsilon \rightarrow 0$,

$$
W_{0}^{\varepsilon}(e) \rightarrow W_{0}(e)
$$

for all $e$ as $\varepsilon \rightarrow 0$. In addition $W_{0}^{\varepsilon}$ must satisfy the following regularity property,

$$
\left|W_{0}^{\varepsilon}\left(e_{1}\right)-W_{0}^{\varepsilon}\left(e_{2}\right)\right| \leq \beta\left(1+\left|e_{1}\right|^{p-1}+\left|e_{2}\right|^{p-1}\right)\left|e_{1}-e_{2}\right|
$$

for all $e_{1}, e_{2}$, which, if $W_{0}^{\varepsilon}$ is smooth, is equivalent to

$$
\left|\nabla W_{0}^{\varepsilon}(e)\right| \leq C\left(1+|e|^{p-1}\right) .
$$

Obviously, due to the convergence (5), $W_{0}$ possesses the same regularity property:

$$
\left|W_{0}\left(e_{1}\right)-W_{0}\left(e_{2}\right)\right| \leq \beta\left(1+\left|e_{1}\right|^{p-1}+\left|e_{2}\right|^{p-1}\right)\left|e_{1}-e_{2}\right| .
$$




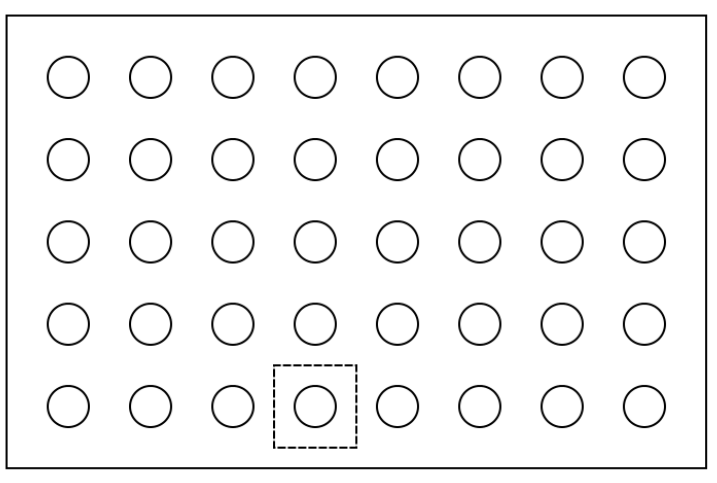

$\varepsilon Y=\varepsilon Y_{0} U \varepsilon Y_{1}$

Fig. 1 Periodic composite.

Let $\Omega$ be a bounded domain in $\mathbb{R}^{n}, n \geq 2$, with a Lipschitz boundary. Let $Y:=[0,1)^{n}$ be a reference cell, $Y_{0}$ be a Lipschitz domain such that $\bar{Y}_{0} \subset(0,1)^{n}$, and $Y_{1}:=Y \backslash \bar{Y}_{0}$. Denote by $E_{0}$ a $Y$-periodic set $\bigcup_{k \in \mathbb{Z}^{n}}\left(Y_{0}+k\right)$ and $E_{1}:=\mathbb{R}^{n} \backslash \bar{E}_{0}$. We describe the "soft" component $E_{0}^{\mathcal{E}}$ of an elastic composite as the union of all small sets $\varepsilon\left(Y_{0}+z\right), z \in \mathbb{Z}^{n}$, such that $\varepsilon(Y+z) \cap\left(\mathbb{R}^{n} \backslash \Omega\right)=\varnothing$. Further, the "stiff" component is defined as $E_{1}^{\varepsilon}=\Omega \backslash \bar{E}_{0}^{\varepsilon}$. Denote by $\chi_{i}^{\varepsilon}(x)$ the characteristic functions of the sets $E_{i}^{\mathcal{E}}, i=1,2$. We will study the asymptotic properties of the following high-contrast sequence of integral functionals ( $c f .(2))$ :

$$
I^{\varepsilon}(u):=\int_{\Omega}\left(W_{1}(\nabla u(x)) \chi_{1}^{\varepsilon}(x)+W_{0}^{\varepsilon}(\varepsilon \nabla u(x)) \chi_{0}^{\varepsilon}(x)\right) \mathrm{d} x .
$$

Notice that we can drop the body force term $-\int_{\Omega} u(x) \cdot f(x) \mathrm{d} x$ in (2), due to the obvious additivity property of $\Gamma$-convergence with respect to the linear terms.

It is clear from the form of the functionals $I^{\varepsilon}$ that as $\varepsilon \rightarrow 0$, on the set $E_{0}^{\varepsilon}$ much larger values of the gradient $\nabla u$ are allowed than on $E_{1}^{\varepsilon}$. If we speak about elasticity $(n \times m=3 \times 3)$ this means that the elastic material in $E_{0}^{\varepsilon}$ is much softer (allows larger deformations) than the material in $E_{1}^{\varepsilon}$. Therefore we will use the terms "soft component" and "stiff component" (the latter is often termed "matrix" in the literature on high-contrast problems) when referring to the sets $E_{0}^{\mathcal{E}}$ and $E_{1}^{\mathcal{\varepsilon}}$ respectively.

We reserve the notation $C$ for a generic positive constant independent of $\varepsilon$, whose specific value may change from one place to another within the paper or even within a single sequence of relations. Throughout the paper we assume that any function of two variables $f(x, y)$ defined on $\Omega \times Y$ is extended periodically with respect to $y$ into $\Omega \times \mathbb{R}^{n}$, so that the formal substitution $y=x / \varepsilon$ will make sense for any $x \in \Omega$.

By $Q W_{0}$ we denote the quasiconvex envelope of $W_{0}$, see $e$.g. [17]. If $V_{1} \geq V_{2}$ then for their quasiconvex envelopes the inequality is preserved, $Q V_{1} \geq Q V_{2}$. Since

\footnotetext{
2 A slightly more general growth condition from below than putting, for example, $\alpha|e|^{p}$, is used in order to accommodate some natural physical requirements, such as frame indifference and zero elastic energy density for a "rigid motion" regime (when one has to take $c>n$ ), $c f$. Remark 2 below.
} 
the functions $\alpha|e|^{p}$ and $\beta\left(1+|e|^{p}\right)$ are quasiconvex and the quasiconvex envelope of a quasiconvex function equals itself, the quasiconvex envelope $Q W_{0}$ satisfies the standard growth conditions.

Remark 2 There is a reason for taking a sequence $W_{0}^{\varepsilon}$ rather than a fixed $W_{0}$ for describing the soft component of the material. Two natural physical requirements for the stored-energy function is that it must be frame indifferent and attain its minimum value at $e=\nabla u=I$. However, in the case of a soft-component stored-energy function $W_{0}$ that is independent of $\varepsilon$ these requirements would imply $W_{0}(\varepsilon R)=0=\min W_{0}$ for any $\varepsilon>0$ and any rotation $R$, which is too restrictive, while our setting can easily accommodate these assumptions. For instance, if $W_{0}^{\varepsilon}$ is given by the formula

$$
W_{0}^{\varepsilon}(e)=\widehat{W}\left((1-\varepsilon) I+\sqrt{e^{T} e}\right),
$$

which for the scaled gradient takes the form

$$
W_{0}^{\varepsilon}(\varepsilon \nabla u)=\widehat{W}\left(I+\varepsilon\left(\sqrt{(\nabla u)^{T} \nabla u}-I\right)\right),
$$

where $\widehat{W}(e)$ is a continuous function satisfying the standard growth conditions and attaining its minimum at $e=I, \widehat{W}(I)=0$, then $W_{0}^{\varepsilon}(\varepsilon \nabla u)$ is frame indifferent $\left(W_{0}^{\varepsilon}(\varepsilon R \nabla u)=W_{0}^{\varepsilon}(\varepsilon \nabla u)\right.$ for any rotation $\left.R\right)$ and attains its minimum value at $\nabla u=I, W_{0}^{\varepsilon}(\varepsilon I)=\widehat{W}(I)=0$. Note that for any sequence $e_{\varepsilon} \rightarrow e$ as $\varepsilon \rightarrow 0$ there is convergence

$$
W_{0}^{\varepsilon}\left(e_{\varepsilon}\right) \rightarrow \widehat{W}\left(I+\sqrt{e^{T} e}\right)
$$

as $\varepsilon \rightarrow 0$, in particular, (5) is satisfied with $W_{0}(e)=\widehat{W}\left(I+\sqrt{e^{T} e}\right)$.

\section{Preliminaries: $\Gamma\left(L^{p}\right)$-convergence, two-scale convergence and an extension lemma}

In what follows (see Section 4) we discuss the standard $\Gamma\left(L^{p}\right)$-convergence, see [8], applied to the present high-contrast setting. Here we recall its definition and its most important property implying convergence of the infima of $\Gamma$-convergent functionals to the minimum of their $\Gamma$-limit and convergence of an infimising sequence to a minimiser of the $\Gamma$-limit. We also give a definition of two-scale convergence and formulate an extension lemma, which we make use of in the subsequent sections.

Definition 3 Let $F_{j}: L^{p}(\Omega) \rightarrow \overline{\mathbb{R}}$ be a sequence of functionals and $F_{\infty}: L^{p}(\Omega) \rightarrow$ $\overline{\mathbb{R}}$, where $\Omega \in \mathbb{R}^{n}$ is bounded and open, and $\overline{\mathbb{R}}:=\mathbb{R} \cup+\infty$. We say that $F_{j} \Gamma\left(L^{p}\right)$ converges to $F_{\infty}$, and write $\Gamma\left(L^{p}\right)-\lim _{j} F_{j}=F_{\infty}$, if for all $u \in L^{p}(\Omega)$ we have

1. for every sequence $u_{j} \rightarrow u$ in $L^{p}(\Omega)$ one has

$$
F_{\infty}(u) \leq \liminf _{j} F_{j}\left(u_{j}\right)
$$


2. there exists a sequence $u_{j} \rightarrow u$ in $L^{p}(\Omega)$ such that

$$
F_{\infty}(u)=\lim _{j} F_{j}\left(u_{j}\right) \text {. }
$$

Remark 4 As is customary in the theory of $\Gamma$-convergence, whenever there is a sequence of functionals $F_{\varepsilon}$ parametrised by a "continuous" parameter $\varepsilon \in\left(0, \varepsilon_{0}\right]$, we write $\Gamma\left(L^{p}\right)-\lim _{\varepsilon \rightarrow 0} F_{\varepsilon}=F_{0}$ if for any sequence $\varepsilon_{j} \rightarrow 0$ one has $\Gamma\left(L^{p}\right)-\lim _{j} F_{\varepsilon_{j}}=$ $F_{0}$.

The fundamental requirement for a proper variational convergence is that it must provide the convergence of infima and infimising sequences for $F_{j}$ to the minimum and a minimiser of the limiting functional $F_{\infty}$. The $\Gamma$-convergence provides the desired goals given that an additional condition is fulfilled, namely, an appropriate compactness property of infimising sequences. This observation is formulated in the following statement, which is a simple consequence of the definition of $\Gamma\left(L^{p}\right)$-convergence.

Theorem 5 Let $F_{j}$ be a sequence of functionals on $L^{p}(\Omega)$ and suppose that there exists a compact set $K \in L^{p}(\Omega)$ such that $\inf _{L^{p}(\Omega)} F_{j}=\inf _{K} F_{j}$ for any $j$. Let $F_{\infty}=\Gamma\left(L^{p}\right)-\lim _{j} F_{j}$ then

$$
\exists \min _{L^{p}(\Omega)} F_{\infty}=\lim _{j} \inf _{L^{p}(\Omega)} F_{j} .
$$

Moreover, if $u_{j} \rightarrow u_{\infty}$ and $\lim _{j} F_{j}\left(u_{j}\right)=\lim _{j} \inf _{L^{p}(\Omega)} F_{j}$, then $u_{\infty}$ is a minimum point for $F_{\infty}$.

The requirement of the existence of a compact $K$ on which the infima for all $j$ are attained ("mild equicoercivity" condition in [8]) can be replaced by a weaker one, namely that there exists a compact sequence $u_{j}$ such that $\liminf _{j} F_{j}\left(u_{j}\right)=$ $\liminf _{j}\left(\inf _{L^{p}(\Omega)} F_{j}\right)$.

Next, we give the definition of two-scale convergence of functions. Using the latter will simplify some of the reasoning below. Two-scale convergence will also be essentially employed in the subsequent sections when dealing with two-scale $\Gamma$-convergence.

Definition 6 We say that a bounded in $L^{p}(\Omega)$ sequence $v_{\varepsilon}$ weakly two-scale converges to a function $v \in L^{p}(\Omega \times Y), v_{\varepsilon}(x) \stackrel{2}{\rightarrow} v(x, y)$, if

$$
\lim _{\varepsilon \rightarrow 0} \int_{\Omega} v_{\varepsilon}(x) \varphi(x) b\left(\frac{x}{\varepsilon}\right) \mathrm{d} x=\int_{\Omega} \int_{Y} v(x, y) \varphi(x) b(y) \mathrm{d} y \mathrm{~d} x
$$

for all $\varphi \in C_{0}^{\infty}(\Omega)$ and all $b \in C_{\text {per }}^{\infty}(Y)$ (where $C_{\text {per }}^{\infty}(Y)$ is the set of $Y$-periodic functions from $C^{\infty}\left(\mathbb{R}^{n}\right)$ ).

Definition 7 We say that a bounded in $L^{p}(\Omega)$ sequence $u_{\varepsilon}$ strongly two-scale converges to a function $u \in L^{p}(\Omega \times Y), u_{\varepsilon}(x) \stackrel{2}{\rightarrow} u(x, y)$, if

$$
\lim _{\varepsilon \rightarrow 0} \int_{\Omega} u_{\varepsilon}(x) v_{\varepsilon}(x) \mathrm{d} x=\int_{\Omega} \int_{Y} u(x, y) v(x, y) \mathrm{d} y \mathrm{~d} x
$$

for all $v_{\varepsilon}(x) \stackrel{2}{\rightarrow} v(x, y)$ from $L^{q}(\Omega)$ and $L^{q}(\Omega \times Y)$ respectively. 
Two-scale convergence, unlike the usual $L^{p}$-convergence, allows one to preserve information about the behaviour of a sequence of functions at the $\varepsilon$-scale when passing to the limit. In this paper we will use some well-known properties of two-scale convergence. The related statements as well as some deeper results can be found e.g. in [37].

Finally, throughout the paper we make use of the following extension lemma $\left(c f .[8]^{3}\right)$

Lemma 8 There exists a linear continuous extension operator $T_{\varepsilon}: W^{1, p}\left(E_{1}^{\varepsilon}\right) \rightarrow$ $W^{1, p}(\Omega)$ and a constant $C>0$ such that

$$
\begin{gathered}
T_{\varepsilon} u=u \text { in } E_{1}^{\varepsilon} \\
\int_{\Omega}\left|T_{\varepsilon} u\right|^{p} \mathrm{~d} x \leq C \int_{E_{1}^{\varepsilon}}|u|^{p} \mathrm{~d} x, \\
\int_{\Omega}\left|\nabla\left(T_{\varepsilon} u\right)\right|^{p} \mathrm{~d} x \leq C \int_{E_{1}^{\varepsilon}}|\nabla u|^{p} \mathrm{~d} x
\end{gathered}
$$

where the constants $C$ does not depend on $\varepsilon$ or $\Omega$.

\section{$4 \Gamma\left(L^{p}\right)$-compactness and its failure in high-contrast homogenisation}

In the present section we discuss compactness of the sequence of high-contrast functionals (8) in the sense of $\Gamma\left(L^{p}\right)$-convergence, providing an explicit formula for the limit functional in a concrete but quite general case. At the end of the section we will give a simple example illustrating that the lack of compactness for infimising sequences leads to the failure of $\Gamma$-convergence to provide a proper variational limit for the related functionals.

It is well known that the $\Gamma$-compactness result (i.e. existence of a $\Gamma$-convergent subsequence) holds for integral functionals of the form

$$
F_{\varepsilon}(u)=\int_{\Omega} f_{\varepsilon}(x, \nabla u) \mathrm{d} x
$$

with a positive stored-energy function $f_{\varepsilon}$ satisfying the growth estimate

$$
\alpha|e|^{p} \leq f_{\varepsilon}(x, e) \leq \beta\left(1+|e|^{p}\right)
$$

see e.g. [8]. It can also be shown that the result holds ${ }^{4}$ for functionals (8). We formulate it in the following statement.

\footnotetext{
${ }^{3}$ Lemma 8 provides somewhat stronger estimates than the extension result of [8], due to the restrictions we impose on the geometry of the set $E_{1}^{\varepsilon}$ near the boundary of $\Omega$, and is derived directly from Lemma B.6 of [8].

4 By a slight variation of the proof of Proposition 12.2 in [8] one can see that the class (10) can be widened to include $f_{\varepsilon}$ that satisfy $\alpha_{\varepsilon}(x)\left(-c+|e|^{p}\right) \leq f_{\varepsilon}(x, e) \leq \beta_{\varepsilon}(x)\left(1+|e|^{p}\right)$, where positive functions $\alpha_{\varepsilon}, \beta_{\varepsilon} \in L^{\infty}(\Omega)$ are such that $1 \leq \frac{\beta_{\varepsilon}}{\alpha_{\varepsilon}} \leq C$ pointwise.
} 
Theorem 9 Let $I^{\varepsilon}$ be given by (8). For every positive sequence $\varepsilon_{i} \rightarrow 0$ there exists a subsequence $\varepsilon_{i_{k}}$ and a measurable function $\varphi \geq 0$ such that

$$
I^{0}(u):=\int_{\Omega} \varphi(\nabla u) \mathrm{d} x=\Gamma\left(L^{p}\right)-\lim _{k \rightarrow \infty} I^{\varepsilon_{i}}(u)
$$

for all $u \in W^{1, p}(\Omega)$ and all open subsets $U$ of $\Omega$.

Given an additional assumption on $W_{0}$ it is even possible to write an explicit formula for $I^{0}$, as follows.

Theorem 10 Let $W_{0}(e)$ in (5) be such that it attains its minimum at $e=0$. Then

$$
I^{0}(u)=\Gamma\left(L^{p}\right)-\lim _{\varepsilon \rightarrow 0} I^{\varepsilon}(u)=\int_{\Omega} W_{1}^{\mathrm{hom}}(\nabla u) \mathrm{d} x+\left|\Omega \times Y_{0}\right| W_{0}(0),
$$

where

$$
W_{1}^{\text {hom }}(e)=\liminf _{t \rightarrow \infty}\left\{\frac{1}{t^{n}} \int_{(0, t)^{n} \cap E_{1}} W_{1}(e+\nabla \varphi) \mathrm{d} x: \varphi \in W_{0}^{1, p}\left((0, t)^{n}\right)\right\} .
$$

Proof Notice that the "stiff part" of the $\Gamma$-limit (11) is exactly the $\Gamma\left(L^{p}\right)$-limit for a non-high-contrast integral functional defined on the perforated domain $E_{1}^{\varepsilon}$ ("connected media", see [8, Part IV]),

$$
I_{1}^{\varepsilon}(u):=\int_{\Omega} W_{1}(\nabla u(x)) \chi_{1}^{\varepsilon}(x) \mathrm{d} x .
$$

Let us fix some $u \in W^{1, p}(\Omega)$ and consider an arbitrary sequence $u_{\varepsilon} \in W^{1, p}(\Omega)$ converging to $u$ in $L^{p}(\Omega)$. There are two possibilities:

1. there exists $C>0$ such that $\left\|\nabla u_{\varepsilon} \chi_{1}^{\varepsilon}\right\|_{L^{p}(\Omega)}+\left\|\varepsilon \nabla u_{\varepsilon} \chi_{0}^{\varepsilon}\right\|_{L^{p}(\Omega)} \leq C$ for small enough $\varepsilon$

2. up to a subsequence $\left\|\nabla u_{\varepsilon} \chi_{1}^{\varepsilon}\right\|_{L^{p}(\Omega)}+\left\|\varepsilon \nabla u_{\varepsilon} \chi_{0}^{\varepsilon}\right\|_{L^{p}(\Omega)} \rightarrow \infty$ as $\varepsilon \rightarrow 0$;

Clearly, in the latter case (case 2) we have $I^{\varepsilon}\left(u_{\varepsilon}\right) \rightarrow \infty$ due to the standard growth conditions. Let us consider the first possibility (case 1). For any $\varepsilon$ there exists an extension $\widetilde{u}_{\varepsilon}$ of the function $u_{\varepsilon} \chi_{1}^{\varepsilon}$ to the whole $\Omega$ such that

$$
\left\|\nabla \widetilde{u}_{\varepsilon}\right\|_{L^{p}(\Omega)} \leq C\left\|\nabla u_{\varepsilon} \chi_{1}^{\varepsilon}\right\|_{L^{p}(\Omega)},
$$

where $C$ does not depend on $\varepsilon$, see Lemma 8. Then $\nabla \widetilde{u}_{\varepsilon}$ is bounded in $L^{p}(\Omega)$. Hence $\widetilde{u}_{\varepsilon}$ is weakly compact in $W^{1, p}(\Omega)$ and strongly compact in $L^{p}(\Omega)$, and there exists $\widetilde{u} \in W^{1, p}(\Omega)$ such that $\widetilde{u}_{\varepsilon} \rightarrow \widetilde{u}$ in $L^{p}(\Omega)$ up to a subsequence. By the properties of two-scale convergence we have

$$
\begin{aligned}
& \widetilde{u}_{\varepsilon} \chi_{1}^{\varepsilon} \stackrel{2}{\rightarrow} \widetilde{u}(x) \chi_{1}(y), \\
& u_{\varepsilon} \chi_{1}^{\varepsilon} \stackrel{2}{\rightarrow} u(x) \chi_{1}(y) .
\end{aligned}
$$

Since $\widetilde{u}_{\varepsilon} \chi_{1}^{\varepsilon}=u_{\varepsilon} \chi_{1}^{\varepsilon}$ by definition, we conclude that $\widetilde{u}(x)=u(x)$. Due to estimate (13), $\varepsilon \nabla \widetilde{u}_{\varepsilon} \rightarrow 0$ in $L^{p}(\Omega)$. This implies the convergence of the soft component integral on $\widetilde{u}_{\varepsilon}$ :

$$
\lim _{\varepsilon \rightarrow 0} \int_{\Omega} W_{0}^{\varepsilon}\left(\varepsilon \nabla \widetilde{u}_{\varepsilon}(x)\right) \chi_{0}^{\varepsilon} \mathrm{d} x=\left|\Omega \times Y_{0}\right| W_{0}(0) .
$$


Equality (14) follows from the dominated convergence theorem in view of the standard growth conditions. Thus we have shown that for any sequence $u_{\varepsilon} \rightarrow u$ in $L^{p}(\Omega)$ possessing the property 1 there exists a sequence $\widetilde{u}_{\varepsilon} \rightarrow u$ in $L^{p}(\Omega)$ such that, firstly,

$$
\begin{aligned}
\liminf _{\varepsilon \rightarrow 0} I^{\varepsilon}\left(\widetilde{u}_{\varepsilon}\right) & \leq \liminf _{\varepsilon \rightarrow 0} I^{\varepsilon}\left(u_{\varepsilon}\right), \\
\limsup _{\varepsilon \rightarrow 0} I^{\varepsilon}\left(\widetilde{u}_{\varepsilon}\right) & \leq \limsup _{\varepsilon \rightarrow 0} I^{\varepsilon}\left(u_{\varepsilon}\right),
\end{aligned}
$$

and, secondly,

$$
\begin{aligned}
\liminf _{\varepsilon \rightarrow 0} I^{\varepsilon}\left(\widetilde{u}_{\varepsilon}\right) & =\liminf _{\varepsilon \rightarrow 0} I_{1}^{\varepsilon}\left(\widetilde{u}_{\varepsilon}\right)+\left|\Omega \times Y_{0}\right| W_{0}(0), \\
\limsup _{\varepsilon \rightarrow 0} I^{\varepsilon}\left(\widetilde{u}_{\varepsilon}\right) & =\limsup _{\varepsilon \rightarrow 0} I_{1}^{\varepsilon}\left(\widetilde{u}_{\varepsilon}\right)+\left|\Omega \times Y_{0}\right| W_{0}(0) .
\end{aligned}
$$

This, using the definition of $\Gamma$-convergence, implies that

$$
\Gamma\left(L^{p}\right)-\lim _{\varepsilon \rightarrow 0} I^{\varepsilon}=\Gamma\left(L^{p}\right)-\lim _{\varepsilon \rightarrow 0} I_{1}^{\varepsilon}+\left|\Omega \times Y_{0}\right| W_{0}(0) .
$$

Combined with the known result (see e.g. [8]) on the $\Gamma\left(L^{p}\right)$-convergence of the sequence $I_{1}^{\varepsilon}$ this yields

$$
\Gamma\left(L^{p}\right)-\lim _{\varepsilon \rightarrow 0} I^{\varepsilon}=I^{0} .
$$

The $\Gamma$-convergence itself without an appropriate compactness property of infimising sequences does not imply the desired convergence of infima of $I^{\varepsilon}$ to the minimum of the $\Gamma\left(L^{p}\right)$-limit. We illustrate this observation in the following example.

Example 11 Let $W_{1}(e)=W_{0}^{\varepsilon}(e)=|e|^{p}$ and consider a one-dimensional minimisation problem for the functional

$$
I^{\varepsilon}(u)=\int_{\Omega}\left(\left|\partial_{x} u\right|^{p} \chi_{1}^{\varepsilon}+\varepsilon^{p}\left|\partial_{x} u\right|^{p} \chi_{0}^{\varepsilon}\right) \mathrm{d} x-\int_{\Omega} f u \mathrm{~d} x,
$$

where $f \in L^{q}(\Omega)$ (recall that $\left.p^{-1}+q^{-1}=1\right)$ is positive and $Y_{0}=(1 / 4,3 / 4)$. Then the $\Gamma\left(L^{p}\right)$-limit for $I^{\varepsilon}$ is given by

$$
I^{0}(u)=\left|Y_{1}\right| \int_{\Omega}\left|\partial_{x} u\right|^{p} \mathrm{~d} x-\int_{\Omega} f u \mathrm{~d} x .
$$

Consider also the functional

$$
\widetilde{I}^{0}(u)=\left|Y_{1}\right| \int_{\Omega}\left|\partial_{x} u_{0}\right|^{p} \mathrm{~d} x+\int_{\Omega} \int_{Y_{0}}\left|\partial_{y} v\right|^{p} \mathrm{~d} y \mathrm{~d} x-\int_{\Omega} \int_{Y} f u \mathrm{~d} y \mathrm{~d} x
$$

acting on functions of the form $u(x, y)=u_{0}(x)+v(x, y)$, where $u_{0} \in W^{1, p}(\Omega)$, $v \in L^{p}\left(\Omega, W_{0}^{1, p}\left(Y_{0}\right)\right)^{5}$. Next we will show that

$$
\inf \widetilde{I}^{0}<\inf I^{0} .
$$

5 By $L^{p}\left(\Omega, W_{0}^{1, p}\left(Y_{0}\right)\right)$ we denote the space of functions defined on $\Omega$ with values in $W_{0}^{1, p}\left(Y_{0}\right)$ equipped with the $L^{p}$-norm. 
Since $\widetilde{I}^{0}(u)=I^{0}\left(u_{0}\right)+G(v)$, where $G(v)=\int_{\Omega} \int_{Y_{0}}\left|\partial_{y} v\right|^{p} \mathrm{~d} y \mathrm{~d} x-\int_{\Omega} \int_{Y_{0}} f v \mathrm{~d} y \mathrm{~d} x$, we only need to show that $\min G(v)<0$. Consider the following function $w \in$ $W_{0}^{1, p}\left(Y_{0}\right)$ extended by zero in $Y_{1}$ :

$$
w(y)=\frac{1}{4}-\left|x-\frac{1}{2}\right| .
$$

Straightforward calculations show that for $\beta>0$

$$
\int_{Y_{0}}\left|\alpha(\beta) \partial_{y} w(y)\right|^{p} \mathrm{~d} y-\beta \int_{Y_{0}} \alpha(\beta) w(y) \mathrm{d} y=-C \beta^{q}<0
$$

where

$$
\begin{gathered}
\alpha(\beta)=\left(\frac{\beta}{8 p}\right)^{q-1}, \\
C=\frac{1-p}{2(8 p)^{q}}>0,
\end{gathered}
$$

Then

$$
\inf G(v) \leq G(\alpha(f(x)) w(y))=-C \int_{\Omega}(f(x))^{p} \mathrm{~d} x<0
$$

since $f(x)$ is positive by the assumption. Hence there exists a function $u=u_{0}(x)+$ $v(x, y)$ such that

$$
\widetilde{I}^{0}(u)<\inf I^{0}
$$

In particular, this function can be chosen such that it is Carathéodory (that is measurable with respect to $x$ and continuous with respect to $y)$ and $v(x, y)$ is differentiable in $x$.

Consider now the sequence $u_{\varepsilon}(x)=u_{0}(x)+v\left(x, \varepsilon^{-1} x\right)$. It follows from the properties of the two-scale convergence, see $e . g$. [37], that

$$
\begin{gathered}
u_{\varepsilon} \stackrel{2}{\rightarrow} u, \\
\left|\partial_{x} u_{\varepsilon}\right|^{p} \chi_{1}^{\varepsilon} \stackrel{2}{\rightarrow}\left|\partial_{x} u_{0}\right|^{p} \chi_{1}, \\
\varepsilon^{p}\left|\partial_{x} u_{\varepsilon}\right|^{p} \chi_{0}^{\varepsilon} \stackrel{2}{\rightarrow}\left|\partial_{y} v\right|^{p} \chi_{0} .
\end{gathered}
$$

This implies the convergence

$$
I^{\varepsilon}\left(u_{\varepsilon}\right) \rightarrow \widetilde{I}^{0}(u)
$$

as $\varepsilon \rightarrow 0$. Then from (15) it follows that

$$
\liminf _{\varepsilon \rightarrow 0} I^{\varepsilon}<\inf I^{0}
$$

The above example ${ }^{6}$ indicates that the appropriate type of variational convergence in the high-contrast setting would have to be capable of accounting for rapid

\footnotetext{
${ }^{6}$ Strictly speaking, the example does not satisfy the connectedness condition on the stiff component $E_{1}^{\varepsilon}$. However, its multi-dimensional analogue, whose construction is clearly similar while somewhat more cumbersome, would comply with all the requirements of Sections 1 and 2 and would still lead to (16), i.e. to the failure of the usual $\Gamma\left(L^{p}\right)$-convergence to pick up the behaviour of minimising sequences for $I^{\varepsilon}$.
} 
oscillations on the soft inclusions "in the very limit" as $\varepsilon \rightarrow 0$. At the same time, we aim at making sure that the requirement of convergence of infima and of minimising sequences is satisfied. As was discussed in Section 3 in the $L^{p}(\Omega)$ setting, one way to ensure this is to ask for these sequences to be compact with respect to the convergence in the underlying function space. It is well-known that the limiting behaviour as $\varepsilon \rightarrow 0$ of sequences $u_{\varepsilon}$ satisfying an a priori bound on $\varepsilon \nabla u_{\varepsilon}$ can be characterised using the notion of two-scale convergence (see Definitions 6 and 7 and [39]). Hence, we extend the usual notion of $\Gamma$-convergence to enable one to handle such sequences within the new framework. The result of this extension is the "two-scale $\Gamma$-convergence", which we introduce in the next section. Some ideas in the direction of such an extension have appeared in the literature in other contexts, see [21], [34], [35]. Our main objective is to describe the behaviour of high-contrast functionals of the form (2), so we focus on those properties of the two-scale $\Gamma$-convergence that are relevant in our setting.

\section{Two-scale $\Gamma$-convergence}

The definition of the two-scale $\Gamma$-convergence (for which we use the shorthand $\Gamma_{\mathrm{ts}}\left(L^{p}\right)$-convergence) is very similar to the definition of the standard $\Gamma$-convergence with the only difference that instead of using the $L^{p}$-topology we use the two-scale convergence of functions.

Definition 12 Let $F_{\varepsilon}: L^{p}(\Omega) \rightarrow \overline{\mathbb{R}}$ be a sequence of functionals and $F_{0}: L^{p}(\Omega \times$ $Y) \rightarrow \overline{\mathbb{R}}$. We say that $F_{\mathcal{\varepsilon}} \Gamma_{\mathrm{ts}}\left(L^{p}\right)$-converges to $F_{0}$ and write $F_{0}=\Gamma_{\mathrm{ts}}\left(L^{p}\right)-\lim _{\mathcal{\varepsilon} \rightarrow 0} F_{\mathcal{\varepsilon}}$ if for all $u \in L^{p}(\Omega \times Y)$ we have

1. for every sequence $L^{p}(\Omega) \ni u_{\varepsilon} \stackrel{2}{\rightarrow} u$ one has

$$
F_{0}(u) \leq \liminf _{\varepsilon \rightarrow 0} F_{\varepsilon}\left(u_{\varepsilon}\right)
$$

2. there exists a sequence $L^{p}(\Omega) \ni u_{\varepsilon} \stackrel{2}{\rightarrow} u$ such that

$$
F_{0}(u)=\lim _{\varepsilon \rightarrow 0} F_{\varepsilon}\left(u_{\varepsilon}\right) .
$$

One of the important properties of the usual $\Gamma$-convergence is that the $\Gamma$-limit is lower semicontinuous with respect to the topology of the underlying function (or, more generally, metric) space. The proof of this fact is simple and based on use of the first property in the definition of $\Gamma$-convergence and the method of extraction of a diagonal subsequence, see e.g. [8, Chapter 7]. In the present scope of the two-scale $\Gamma$-convergence the limit functional also possesses the lower semicontinuity property. However, the proof of this fact is not so obvious due to the lack of metric properties for the two-scale convergence. When $u_{\varepsilon} \rightarrow u$ in $L^{p}(\Omega)$ one can measure "closeness" of $u_{\varepsilon}$ to $u$ by $\left\|u_{\varepsilon}-u\right\|_{L^{p}(\Omega)}$. If $u_{\varepsilon}(x) \stackrel{2}{\rightarrow}$ $u(x, y)$ and $u$ is a Carathéodory function, then one can measure the closeness by $\left\|u_{\varepsilon}(x)-u\left(x, \varepsilon^{-1} x\right)\right\|_{L^{p}(\Omega)}$, but if $u$ is not Carathéodory, i.e. $u\left(x, \varepsilon^{-1} x\right)$ behaves "badly", then there is no obvious way of doing this. Next we address the problem of extracting a diagonal sequence in the scope of two-scale convergence and prove the lower semicontinuity of a two-scale $\Gamma$-limit. 
Whereas the correct passage from the space of two variables to the space of one variable by change $y=x / \varepsilon$ requires some regularity properties of function and not possible in general, the unfolding method (see e.g. [14]) allows the passage in the opposite direction while preserving convergence properties. Below we give the definition of the unfolding operator and its weak convergence property from [14]. We denote by $[y]$ the integer part of the vector $y$ (that is each component of $[y]_{i}$ is the largest integer such that $[y]_{i} \leq y$, thus $y-[y] \in Y$ ).

Definition 13 For Lebesgue-measurable functions $\varphi$ on $\Omega$, the unfolding operator $\mathscr{T}_{\varepsilon}$ is defined as follows:

$$
\mathscr{T}_{\varepsilon}(\varphi)(x, y)=\varphi\left(\varepsilon\left[\frac{x}{\varepsilon}\right]+\varepsilon y\right) \text { for }(x, y) \in \Omega \times Y .
$$

Proposition 14 Let $w_{\varepsilon}$ be a bounded sequence in $L^{p}(\Omega)$. The following statements are equivalent:

1. $w_{\varepsilon} \stackrel{2}{\rightarrow} w \in L^{p}(\Omega \times Y)$,

2. $\mathscr{T}_{\varepsilon}\left(w_{\varepsilon}\right) \rightarrow w$ in $L^{p}(\Omega \times Y)$.

The above proposition immediately implies equivalence of the strong convergence properties.

Proposition 15 A sequence $w_{\varepsilon}$ from $L^{p}(\Omega)$ strongly two-scale converges to $w \in$ $L^{p}(\Omega \times Y), w_{\varepsilon} \stackrel{2}{\rightarrow} w$, if and only if $\mathscr{T}_{\varepsilon}\left(w_{\varepsilon}\right) \rightarrow w$ in $L^{p}(\Omega \times Y)$.

Proof Let $w_{\varepsilon} \stackrel{2}{\rightarrow} w$. Then $w_{\varepsilon} \stackrel{2}{\rightarrow} w,\left\|w_{\varepsilon}\right\|_{L^{p}(\Omega)} \rightarrow\|w\|_{L^{p}(\Omega \times Y)}$ by the properties of the two-scale convergence, in particular $w_{\varepsilon}$ is bounded in $L^{p}(\Omega)$. By Proposition 14

$$
\mathscr{T}_{\varepsilon}\left(w_{\varepsilon}\right) \rightarrow w \text { in } L^{p}(\Omega \times Y) .
$$

Also, by properties of the unfolding operator $\left\|w_{\varepsilon}\right\|_{L^{p}(\Omega)}=\left\|\mathscr{T}_{\mathcal{E}}\left(w_{\varepsilon}\right)\right\|_{L^{p}(\Omega \times Y)}$, hence we have the convergence of norms $\left\|\mathscr{T}_{\mathcal{\varepsilon}}\left(w_{\varepsilon}\right)\right\|_{L^{p}(\Omega \times Y)} \rightarrow\|w\|_{L^{p}(\Omega \times Y)}$. Together with weak convergence (19) the latter implies $\mathscr{T}_{\varepsilon}\left(w_{\varepsilon}\right) \rightarrow w$ in $L^{p}(\Omega \times Y)$. The proof of the opposite statement is analogous.

Remark 16 The above proposition ensures the metric properties of the strong twoscale convergence. Namely, $w_{\varepsilon} \stackrel{2}{\rightarrow} w$ if and only if $\lim _{\mathcal{E} \rightarrow 0}\left\|\mathscr{T}_{\varepsilon}\left(w_{\varepsilon}\right)-w\right\|_{L^{p}(\Omega \times Y)}=$ 0 . Thus $\left\|\mathscr{T}_{\varepsilon}\left(w_{\varepsilon}\right)-w\right\|_{L^{p}(\Omega \times Y)}$ indeed measures a "distance" between $w_{\varepsilon}$ and $w$ at least to the extent to which a distance between elements of different spaces can be measured.

Proposition 17 Let $u^{k}(x, y) \rightarrow u(x, y)$ in $L^{p}(\Omega \times Y)$ and let for any $k \in \mathbb{N}$ a sequence $v_{\varepsilon}^{k} \in L^{p}(\Omega)$ be such that $v_{\varepsilon}^{k}(x) \stackrel{2}{\rightarrow} u^{k}(x, y)$ as $\varepsilon \rightarrow 0$. Let $\varepsilon_{k}^{\prime}$ be some given positive sequence. Then there exists a sequence $\varepsilon_{k} \rightarrow 0$, with the property $\varepsilon_{k} \leq \varepsilon_{k}^{\prime}$, and such that $v_{\varepsilon_{k}}^{k}(x) \stackrel{2}{\rightarrow} u(x, y)$.

Proof Proposition 15 makes the proof elementary. Let us apply the unfolding operator to the sequences $v_{\varepsilon}^{k}$. We have $\mathscr{T}_{\varepsilon}\left(v_{\varepsilon}^{k}\right) \rightarrow u^{k}(x, y)$ in $L^{p}(\Omega \times Y)$ for any $k$. One can extract a diagonal subsequence $\mathscr{T}_{\varepsilon_{k}}\left(v_{\varepsilon_{k}}^{k}\right)$ (with $\varepsilon_{k} \rightarrow 0$ and $\varepsilon_{k} \leq \varepsilon_{k}^{\prime}$ ) converging to $u$ in $L^{p}(\Omega \times Y)$. Then by Proposition 15 we obtain $v_{\varepsilon_{k}}^{k}(x) \stackrel{2}{\rightarrow} u(x, y)$. 
Theorem 18 Let $F_{0}=\Gamma_{\mathrm{ts}}\left(L^{p}\right)-\lim _{\varepsilon \rightarrow 0} F_{\varepsilon}$. Then $F_{0}$ is lower semicontinuous with respect to the strong convergence in $L^{p}(\Omega \times Y)$.

Proof Let $u^{k} \rightarrow u$ in $L^{p}(\Omega \times Y)$. By (18) for each $k \in \mathbb{N}$ there exists a sequence $v_{\varepsilon}^{k}(x) \stackrel{2}{\rightarrow} u^{k}(x, y)$ such that $\lim _{\mathcal{E} \rightarrow 0} F_{\varepsilon}\left(v_{\varepsilon}^{k}\right)=F_{0}\left(u^{k}\right)$. Let a sequence $\varepsilon_{k}^{\prime}$ be chosen such that for every $k$

$$
\left|F_{\varepsilon}\left(v_{\varepsilon}^{k}\right)-F_{0}\left(u^{k}\right)\right| \leq 1 / k
$$

as soon as $\varepsilon \leq \varepsilon_{k}^{\prime}$. By Proposition 17 there is a sequence $\varepsilon_{k}$, $\varepsilon_{k} \leq \varepsilon_{k}^{\prime}$, with the property that $v_{\varepsilon_{k}}^{k} \stackrel{2}{\rightarrow} u$. Then by (17) and (20) we have

$$
F_{0}(u) \leq \liminf _{k \rightarrow \infty} F_{\varepsilon_{k}}\left(v_{\varepsilon_{k}}^{k}\right)=\liminf _{k \rightarrow \infty} F_{0}\left(u^{k}\right) .
$$

The definition of $\Gamma_{\mathrm{ts}}\left(L^{p}\right)$-convergence is quite general, it gives no information even on the structure of the function space on which the limit functional is defined (i.e. attains finite values). In order to understand this we need to consider a sequence of functions with bounded energy and pass to the limit. Let a sequence $u_{\varepsilon}$ be bounded in $L^{p}(\Omega)$ and be a bounded energy sequence for the sequence of functionals $I^{\varepsilon}$, i.e. such that $I^{\varepsilon}\left(u_{\varepsilon}\right) \leq C$. The standard growth conditions (4) imply that

$$
\left\|\nabla u_{\varepsilon} \chi_{1}^{\varepsilon}\right\|_{L^{p}(\Omega)}+\left\|\varepsilon \nabla u_{\varepsilon} \chi_{0}^{\varepsilon}\right\|_{L^{p}(\Omega)} \leq C .
$$

By the compactness property of two-scale convergence (see e.g. $[39]^{7}$ ) we have

$$
\begin{gathered}
u_{\varepsilon} \stackrel{2}{\rightarrow} u(x, y) \in L^{p}\left(\Omega, W_{\mathrm{per}}^{1, p}(Y)\right), \\
\varepsilon \nabla u_{\varepsilon} \stackrel{2}{\rightarrow} \nabla_{y} u(x, y),
\end{gathered}
$$

where $W_{\text {per }}^{1, p}(Y)$ is the Sobolev space of functions periodic on $Y$. Clearly, the characteristic function $\chi_{1}^{\varepsilon}(x)$ strongly two-scale converges to $\chi_{1}(y)$. Hence, by properties of two-scale convergence

$$
\begin{gathered}
\chi_{1}^{\varepsilon} u_{\varepsilon} \stackrel{2}{\rightarrow} \chi_{1}(y) u(x, y), \\
\varepsilon \chi_{1}^{\varepsilon} \nabla u_{\varepsilon} \stackrel{2}{\rightarrow} \chi_{1}(y) \nabla_{y} u(x, y) .
\end{gathered}
$$

Then from $\left\|\varepsilon \chi_{1}^{\varepsilon} \nabla u_{\varepsilon}\right\|_{L^{p}(\Omega)} \rightarrow 0\left(c f\right.$. (21)) in follows that $\chi_{1}(y) \nabla_{y} u(x, y) \equiv 0$, that is $u(x, y)$ does not depend on $y$ when $y \in Y_{1}$,

$$
\chi_{1}(y) u(x, y)=\chi_{1}(y) u_{0}(x) .
$$

This observation allows to represent the limit function in the form

$$
u(x, y)=u_{0}(x)+v(x, y),
$$

where $v(x, y) \in L^{p}\left(\Omega, W_{0}^{1, p}\left(Y_{0}\right)\right)$.

\footnotetext{
7 The corresponding statement in [39] is proven for the case $L^{2}$. However, it remains valid for the general $L^{p}$, the related proof being identical.
} 
Next we show that $u_{0}(x) \in W^{1, p}(\Omega)$. We denote an extension of $u_{\varepsilon}$ by Lemma 8 by $\widetilde{u}_{\varepsilon}$, let also $v_{\varepsilon}=u_{\varepsilon}-\widetilde{u}_{\varepsilon}$. Lemma 8 and (21) imply that the sequence $\widetilde{u}_{\varepsilon}$ is bounded in $W^{1, p}(\Omega)$, and, hence, converges to some $\widetilde{u}_{0} \in W^{1, p}(\Omega)$ strongly in $L^{p}(\Omega)$ (at least up to a subsequence). By the properties of two-scale convergence we have

$$
\chi_{1}^{\varepsilon} u_{\varepsilon}=\chi_{1}^{\varepsilon} \widetilde{u}_{\varepsilon} \stackrel{2}{\rightarrow} \chi_{1}(y) \widetilde{u}_{0}(x) .
$$

Comparing the latter to (22), (23) we conclude that $u_{0}=\widetilde{u}_{0} \in W^{1, p}(\Omega)$.

We next split the functional $I^{\varepsilon}$ in two terms corresponding to the stiff and soft components,

$$
I^{\varepsilon}(u)=I_{1}^{\varepsilon}+I_{0}^{\varepsilon}:=\int_{\Omega} W_{1}(\nabla u) \chi_{1}^{\varepsilon} \mathrm{d} x+\int_{\Omega} W_{0}^{\varepsilon}(\varepsilon \nabla u) \chi_{0}^{\varepsilon} \mathrm{d} x
$$

and consider each term separately when passing to the limit as $\varepsilon \rightarrow 0$. Finding the $\Gamma_{\mathrm{ts}}\left(L^{p}\right)$-limit of the "stiff" component $I_{1}^{\varepsilon}$ is straightforward due to the fact that its $\Gamma_{\mathrm{ts}}\left(L^{p}\right)$-limit coincides with the $\Gamma\left(L^{p}\right)$-limit of the perforated media functional studied in [8]. On the contrary, derivation of the $\Gamma_{\mathrm{ts}}\left(L^{p}\right)$-limit of the "soft" component $I_{0}^{\varepsilon}$ is non-trivial, it involves using properties of two-scale convergence, periodic unfolding method, properties of quasiconvexity and multi-scale analysis. The basic idea is that the argument $\varepsilon \nabla u_{\varepsilon}$ of $I_{0}^{\varepsilon}$ can be written as $\varepsilon \nabla \widetilde{u}_{\varepsilon}+\varepsilon \nabla v_{\varepsilon}$, where the first term converges to zero in the $L^{p}$-norm ( $c f$. the discussion above), it allows us to consider $I_{0}^{\varepsilon}$ on the family of sequences $v_{\mathcal{\varepsilon}} \in W_{0}^{1, p}\left(E_{0}^{\varepsilon}\right)$ strongly twoscale converging to elements of $L^{p}\left(\Omega, W_{0}^{1, p}\left(Y_{0}\right)\right)$. Then we will show that passing to the limit on the stiff and soft components separately and then summing up the limits yields the correct $\Gamma_{\mathrm{ts}}\left(L^{p}\right)$-limit for the full problem. In the next section we implement the first step in the described strategy.

\section{Two-scale $\Gamma$-limit of the stiff component}

Our focus in the present work is on extending the results of [37], [9], [41], in terms of deriving two-scale limit problems (as $\varepsilon \rightarrow 0$ ) for a general class of high-contrast integral functionals. An essential element of the related settings is the presence of a main, "matrix", material, whose properties retain the classical, non-contrast behaviour in all directions at each point of the ambient space. This implies, in particular, that, once a certain extension procedure is carried out, the passing to the limit can be treated separately on the "matrix" ("stiff") and on the "inclusions" ("soft"). In the present section we determine the "stiff" part of the two-scale limit, which does not present any substantial technical challenges, apart from the need to justify the procedure of splitting the functional into the "stiff" and "soft" components (see Section 8).

We consider the stiff part of the functional, $I_{1}^{\varepsilon}=\int_{\Omega} W_{1}(\nabla u) \chi_{1}^{\varepsilon} \mathrm{d} x$, defined on functions from $W^{1, p}\left(E_{1}^{\varepsilon}\right)$. Let a bounded in $L^{p}\left(E_{1}^{\varepsilon}\right)$ sequence $u_{\varepsilon} \in W^{1, p}\left(E_{1}^{\varepsilon}\right)$ be a bounded energy sequence for $I_{1}^{\varepsilon}$. Analogously to the above, one can show that its extension $\widetilde{u}_{\varepsilon}$ by Lemma 8 converges to some $u \in W^{1, p}(\Omega)$ strongly in $L^{p}(\Omega)$ up to a subsequence. At the same time, $u_{\varepsilon} \chi_{1}^{\varepsilon}=\widetilde{u}_{\varepsilon} \chi_{1}^{\varepsilon} \stackrel{2}{\rightarrow} u(x) \chi_{1}(y)$. Thus the $\Gamma_{\mathrm{ts}}\left(L^{p}\right)$ convergence of $I_{1}^{\varepsilon}$ is equivalent to its $\Gamma\left(L^{p}\right)$-convergence in the sense of [8], and the following theorem ([24], [7]) is valid. 
Theorem 19 Let the stored-energy function $W_{1}$ satisfy the conditions of Section 2. Then the two-scale $\Gamma$-limit of the functional $I_{1}^{\varepsilon}$ is given by

$$
I_{1}^{\mathrm{hom}}(u):=\Gamma_{\mathrm{ts}}\left(L^{p}\right)-\lim _{\varepsilon \rightarrow 0} I_{1}^{\varepsilon}(u)=\int_{\Omega} W_{1}^{\mathrm{hom}}(\nabla u) \mathrm{d} x,
$$

where

$$
W_{1}^{\text {hom }}(e)=\liminf _{t \rightarrow \infty}\left\{\frac{1}{t^{n}} \int_{(0, t)^{n} \cap E_{1}} W_{1}(e+\nabla \varphi) \mathrm{d} x: \varphi \in W_{0}^{1, p}\left((0, t)^{n}\right)\right\},
$$

and $u \in W^{1, p}(\Omega)$.

\section{Two-scale $\Gamma$-limit of the soft component}

7.1 Lower semi-continuity type result

In this section we will consider the "soft inclusions functional" (cf. (24))

$$
I_{0}^{\varepsilon}(u)=\int_{\Omega} W_{0}^{\varepsilon}(\varepsilon \nabla u) \chi_{0}^{\varepsilon} \mathrm{d} x
$$

defined on functions from $W_{0}^{1, p}\left(E_{0}^{\varepsilon}\right)$. We will need the following auxiliary lemma, which is very similar to the one in [17] where it serves as a key step for the proof of the lower semicontinuity property of quasiconvex functionals. (We use the notation " $\rightarrow$ " and " $\rightarrow$ " for weak and strong convergence in corresponding functional spaces.)

Lemma 20 Let $D, G \in \mathbb{R}^{n}$ be cubes with edges parallel to the axes, $e \in \mathbb{R}^{n \times m}$ and $f(e)$ be quasiconvex in $e$ and satisfy the standard growth condition. Let a sequence $z_{\varepsilon}=z_{\varepsilon}(x, y) \in L^{p}\left(D, W^{1, p}(G)\right)$ be such that $\nabla_{y} z_{\varepsilon}(x, y) \rightarrow 0$ and $z_{\varepsilon} \rightarrow 0$ in $L^{p}(D \times G)$. Then

$$
\liminf _{\varepsilon \rightarrow 0} \int_{D \times G} f\left(e+\nabla_{y} z_{\varepsilon}\right) \mathrm{d} y \mathrm{~d} x \geq \int_{D \times G} f(e) \mathrm{d} y \mathrm{~d} x
$$

Proof Let $K$ be an integer and let $G_{0} \subset G_{1} \subset \ldots \subset G_{K} \subset G$ be cubes such that the centres of $G_{k}$ and $G$ coincide for any $k=0,1, \ldots, K$, and

$$
\begin{gathered}
\frac{1}{2} \operatorname{dist}\left(G_{0}, \partial G\right)=R, \\
\operatorname{dist}\left(G_{0}, \partial G_{k}\right)=\frac{k}{K} R,
\end{gathered}
$$

for some $R>0$. Let $\varphi_{k}(y) \in C^{\infty}(G)$ be scalar cut-off functions:

$$
0 \leq \varphi_{k} \leq 1,\left|\nabla \varphi_{k}\right| \leq \widetilde{C} \frac{K}{R}, \varphi_{k}(y)= \begin{cases}1 & \text { if } y \in G_{k-1}, \\ 0 & \text { if } y \in G-G_{k},\end{cases}
$$

with some $\widetilde{C}>0$ independent of $K$ and $R$. Denote

$$
z_{\varepsilon}^{k}=z_{\varepsilon} \varphi_{k} .
$$


Then $z_{\varepsilon}^{k} \in L^{p}\left(D, W_{0}^{1, p}(G)\right)$ and we can use quasiconvexity of $f$ to obtain

$$
\begin{gathered}
\int_{D \times G} f(e) \mathrm{d} y \mathrm{~d} x \leq \int_{D \times G} f\left(e+\nabla_{y} z_{\varepsilon}^{k}\right) \mathrm{d} y \mathrm{~d} x \\
\leq \int_{D \times\left(G-G_{k}\right)} f(e) \mathrm{d} y \mathrm{~d} x+\int_{D \times\left(G_{k}-G_{k-1}\right)} f\left(e+\nabla_{y} z_{\varepsilon}^{k}\right) \mathrm{d} y \mathrm{~d} x \\
+\int_{D \times G_{k-1}} f\left(e+\nabla_{y} z_{\varepsilon}\right) \mathrm{d} y \mathrm{~d} x .
\end{gathered}
$$

The last estimate immediately implies that

$$
\int_{D \times G_{k}} f(e) \mathrm{d} y \mathrm{~d} x \leq \int_{D \times G} f\left(e+\nabla_{y} z_{\varepsilon}\right) \mathrm{d} y \mathrm{~d} x+\int_{D \times\left(G_{k}-G_{k-1}\right)} f\left(e+\nabla_{y} z_{\varepsilon}^{k}\right) \mathrm{d} y \mathrm{~d} x .
$$

We estimate the last term in (28) using the growth assumptions on $f$ and the properties of $\varphi_{k}$, as follows:

$$
\begin{aligned}
& \int_{D \times\left(G_{k}-G_{k-1}\right)} f\left(e+\nabla_{y} z_{\varepsilon}^{k}\right) \mathrm{d} y \mathrm{~d} x \leq \beta \int_{D \times\left(G_{k}-G_{k-1}\right)}\left(1+\left|e+\nabla_{y} z_{\mathcal{\varepsilon}}^{k}\right|^{p}\right) \mathrm{d} y \mathrm{~d} x \\
\leq C \beta & \int_{D \times\left(G_{k}-G_{k-1}\right)}\left(1+|e|^{p}+\left|\varphi_{k} \nabla_{y} z_{\mathcal{\varepsilon}}+\nabla_{y} \varphi_{k} \otimes z_{\mathcal{E}}\right|^{p}\right) \mathrm{d} y \mathrm{~d} x \\
\leq & C \beta \int_{D \times\left(G_{k}-G_{k-1}\right)}\left(1+|e|^{p}+\left|\nabla_{y} z_{\mathcal{\varepsilon}}\right|^{p}+(\widetilde{C} K / R)^{p}\left|z_{\mathcal{E}}\right|^{p}\right) \mathrm{d} y \mathrm{~d} x .
\end{aligned}
$$

Let us take a sum of inequalities (28) for $k$ from 1 to $K$,

$$
\begin{gathered}
f(e) \sum_{k=1}^{K}\left|D \times G_{k}\right| \leq K \int_{D \times G} f\left(e+\nabla_{y} z_{\varepsilon}\right) \mathrm{d} y \mathrm{~d} x \\
+C \int_{D \times\left(G_{K}-G_{0}\right)}\left(1+|e|^{p}+\left|\nabla_{y} z_{\varepsilon}\right|^{p}+(\widetilde{C} K / R)^{p}\left|z_{\varepsilon}\right|^{p}\right) \mathrm{d} y \mathrm{~d} x .
\end{gathered}
$$

Using the fact that $z_{\varepsilon} \rightarrow 0$ in $L^{p}(D \times G)$ yields

$$
\begin{gathered}
f(e) \sum_{k=1}^{K}\left|D \times G_{k}\right| \leq K \liminf _{\varepsilon \rightarrow 0} \int_{D \times G} f\left(e+\nabla_{y} z_{\varepsilon}\right) \mathrm{d} y \mathrm{~d} x \\
+\underset{\varepsilon \rightarrow 0}{\limsup C} \int_{D \times\left(G_{K}-G_{0}\right)}\left(1+|e|^{p}+\left|\nabla_{y} z_{\varepsilon}\right|^{p}\right) \mathrm{d} y \mathrm{~d} x,
\end{gathered}
$$

where the second term on the right hand side is bounded uniformly in $K, R$. Hence dividing (29) by $K$ and letting $K \rightarrow \infty$ and $R \rightarrow 0$ we obtain (27). 
In what follows we will prove the lower semicontinuity type inequality (17) for the soft inclusions functional $I_{0}^{\varepsilon}$.

Lemma 21 Let $v_{\varepsilon}$ be such that $\varepsilon \nabla v_{\varepsilon} \stackrel{2}{\rightarrow} \nabla_{y} v(x, y)$ and $v_{\varepsilon} \stackrel{2}{\rightarrow} v(x, y)$, where $v_{\varepsilon} \in$ $W_{0}^{1, p}\left(E_{0}^{\varepsilon}\right)$ and $v \in L^{p}\left(\Omega, W_{0}^{1, p}\left(Y_{0}\right)\right)$. Then

$$
\liminf _{\varepsilon \rightarrow 0} \int_{\Omega} W_{0}^{\varepsilon}\left(\varepsilon \nabla v_{\varepsilon}\right) \chi_{0}^{\varepsilon} \mathrm{d} x \geq \int_{\Omega} \int_{Y_{0}} Q W_{0}\left(\nabla_{y} v\right) \mathrm{d} y \mathrm{~d} x
$$

where $Q W_{0}$ denotes the quasiconvex envelope of $W_{0}$, i.e. the supremum of all quasiconvex functions not exceeding $W_{0}$.

Proof In this proof we mainly follow [17], essentially employing, however, the multi-scale analysis. First we use the unfolding operator $\mathscr{T}_{\varepsilon}$, see Definition 13, in order to pass to the space of two variables. As it was pointed out in Section 5, an important property of $\mathscr{T}_{\varepsilon}$ is that it preserves the convergence properties of function sequences. Namely, let $w_{\varepsilon}$ be bounded in $L^{p}(\Omega)$, then $w_{\varepsilon}(x) \stackrel{2}{\rightarrow} w(x, y)$ if and only if $\mathscr{T}_{\varepsilon}\left(w_{\varepsilon}\right) \rightarrow w(x, y)$ in $L^{p}(\Omega \times Y)$ and analogously for the strong convergence. $\mathscr{T}_{\varepsilon}$ transforms derivatives with respect to $x$ into the derivatives with respect to $y$ : $\mathscr{T}_{\varepsilon}(\varepsilon \nabla w(x))=\nabla_{y}\left(\mathscr{T}_{\varepsilon}(w)(x, y)\right)$.

Let us denote $\widetilde{v}_{\varepsilon}(x, y)=\mathscr{T}_{\varepsilon}\left(v_{\varepsilon}\right)$. Then $\widetilde{v}_{\varepsilon} \rightarrow v, \nabla_{y} \widetilde{v}_{\varepsilon} \rightarrow \nabla_{y} v$. It is easy to see by a direct calculation that

$$
\int_{\Omega} W_{0}^{\varepsilon}\left(\varepsilon \nabla v_{\varepsilon}\right) \chi_{0}^{\varepsilon} \mathrm{d} x=\int_{\Omega} \int_{Y_{0}} W_{0}^{\varepsilon}\left(\nabla_{y} \widetilde{v}_{\varepsilon}\right) \mathrm{d} y \mathrm{~d} x
$$

Since $W_{0}^{\varepsilon}(e) \geq Q W_{0}^{\varepsilon}(e), \forall e$, and hence,

$$
\int_{\Omega} W_{0}^{\varepsilon}\left(\varepsilon \nabla v_{\varepsilon}\right) \chi_{0}^{\varepsilon} \mathrm{d} x \geq \int_{\Omega} \int_{Y_{0}} Q W_{0}^{\varepsilon}\left(\nabla_{y} \widetilde{v}_{\varepsilon}\right) \mathrm{d} y \mathrm{~d} x
$$

it suffices to show the lower semicontinuity property for the two-scale integral of a quasiconvex function:

$$
\liminf _{\varepsilon \rightarrow 0} \int_{\Omega} \int_{Y_{0}} Q W_{0}^{\varepsilon}\left(\nabla_{y} \widetilde{v}_{\varepsilon}\right) \mathrm{d} y \mathrm{~d} x \geq \int_{\Omega} \int_{Y_{0}} Q W_{0}\left(\nabla_{y} v\right) \mathrm{d} y \mathrm{~d} x .
$$

To this end we cover $\Omega$ by a (finite) union of disjoint cubes $D_{s}$ whose edge length is $h, h>0$, so that $\Omega \subseteq \bigcup_{s} D_{s}$ and $\Omega \cap D_{s} \neq 0, \forall s$. We cover $Y_{0}$ by a union of disjoint cubes $G_{t}$ with the analogous properties. Let us denote by $K_{s t}$ the part of $D_{s} \times G_{t}$ lying in $\Omega \times Y_{0}, K_{s t}:=\left(D_{s} \times G_{t}\right) \cap\left(\Omega \times Y_{0}\right)$. We then take the average of $\nabla_{y} v$ over each $K_{s t}$,

$$
e_{s, t}=\frac{1}{\left|K_{s t}\right|} \int_{K_{s t}} \nabla_{y} v \mathrm{~d} y \mathrm{~d} x
$$

Notice first that

$$
\sum_{s, t} \int_{K_{s t}}\left|\nabla_{y} v-e_{s, t}\right|^{p} \mathrm{~d} y \mathrm{~d} x \rightarrow 0, \text { as } h \rightarrow 0 .
$$


Let us denote

$$
\begin{aligned}
J^{\varepsilon}(w) & =\int_{\Omega} \int_{Y_{0}} Q W_{0}^{\varepsilon}\left(\nabla_{y} w\right) \mathrm{d} y \mathrm{~d} x, \\
J(w) & =\int_{\Omega} \int_{Y_{0}} Q W_{0}\left(\nabla_{y} w\right) \mathrm{d} y \mathrm{~d} x
\end{aligned}
$$

and consider

$$
\begin{gathered}
J^{\varepsilon}\left(\nabla_{y} \widetilde{v}_{\varepsilon}\right)-J\left(\nabla_{y} v\right) \\
=\sum_{s, t} \int_{K_{s t}}\left(Q W_{0}^{\varepsilon}\left(\nabla_{y} v+\left(\nabla_{y} \widetilde{v}_{\varepsilon}-\nabla_{y} v\right)\right)-Q W_{0}^{\varepsilon}\left(e_{s, t}+\left(\nabla_{y} \widetilde{v}_{\varepsilon}-\nabla_{y} v\right)\right)\right) \mathrm{d} y \mathrm{~d} x \\
+\sum_{s, t} \int_{K_{s t}}\left(Q W_{0}^{\varepsilon}\left(e_{s, t}+\left(\nabla_{y} \widetilde{v}_{\varepsilon}-\nabla_{y} v\right)\right)-Q W_{0}^{\varepsilon}\left(e_{s, t}\right)\right) \mathrm{d} y \mathrm{~d} x \\
+\sum_{s, t} \int_{K_{s t}}\left(Q W_{0}^{\varepsilon}\left(e_{s, t}\right)-Q W_{0}\left(e_{s, t}\right)\right) \mathrm{d} y \mathrm{~d} x+\sum_{s, t} \int_{K_{s t}}\left(Q W_{0}\left(e_{s, t}\right)-Q W_{0}\left(\nabla_{y} v\right)\right) \mathrm{d} y \mathrm{~d} x \\
=: J_{1}+J_{2}+J_{3}+J_{4} .
\end{gathered}
$$

The quasiconvex envelope $Q W_{0}^{\varepsilon}$ satisfies the same growth conditions as $W_{0}^{\varepsilon}$ and is rank-one convex (see $e . g$. [17]). Hence it satisfies the same regularity property (see [17, Prop. 2.32]),

$$
\left|Q W_{0}^{\varepsilon}\left(e_{1}\right)-Q W_{0}^{\varepsilon}\left(e_{2}\right)\right| \leq \beta\left(1+\left|e_{1}\right|^{p-1}+\left|e_{2}\right|^{p-1}\right)\left|e_{1}-e_{2}\right|
$$

for any $e_{1}, e_{2} \in \mathbb{R}^{n \times m}$, and analogously for $Q W_{0}$. Now we estimate the term $J_{1}$ as follows. Using (32) and the Hölder inequality yields

$$
\begin{aligned}
&\left|J_{1}\right| \leq \sum_{s, t} \int_{K_{s t}} \beta\left(1+\left|\nabla_{y} \widetilde{v}_{\varepsilon}\right|^{p-1}+\left|e_{s, t}+\left(\nabla_{y} \widetilde{v}_{\varepsilon}-\nabla_{y} v\right)\right|^{p-1}\right)\left|\nabla_{y} v-e_{s, t}\right| \mathrm{d} y \mathrm{~d} x \\
& \leq C \beta\left(\sum _ { s , t } \int _ { K _ { s t } } \left(1+\left|\nabla_{y} \widetilde{v}_{\varepsilon}\right|^{p}+\mid e_{s, t}+\right.\right.\left.\left.\left.\left(\nabla_{y} \widetilde{v}_{\varepsilon}-\nabla_{y} v\right)\right|^{p}\right) \mathrm{~d} y \mathrm{~d} x\right)^{\frac{p-1}{p}} \\
& \times\left(\sum_{s, t} \int_{K_{s t}}\left|\nabla_{y} v-e_{s, t}\right|^{p} \mathrm{~d} y \mathrm{~d} x\right)^{\frac{1}{p}} .
\end{aligned}
$$

Let us fix some $\delta>0$. Since $\nabla_{y} \widetilde{v}_{\varepsilon}$ converges weakly in $L^{p}\left(\Omega \times Y_{0}\right)$ to $\nabla_{y} v$ it is a bounded sequence, hence, the first factor in the last inequality is bounded and (31) yields

$$
\left|J_{1}\right| \leq \delta
$$

for small enough $h$. Analogously,

$$
\left|J_{4}\right| \leq \delta
$$

for small enough $h$. Now applying Lemma 20 we conclude that

$$
\liminf _{\varepsilon \rightarrow 0} J_{2} \geq 0
$$


The term $J_{3}$ vanishes as $\varepsilon \rightarrow 0$ due to the pointwise convergence of $Q W_{0}^{\varepsilon}$ to $Q W_{0}$, which clearly follows from the convergence of $W_{0}^{\varepsilon}$ to $W_{0}$ and the definition of the quasiconvex envelope. Hence we obtain that

$$
\liminf _{\varepsilon \rightarrow 0} J^{\varepsilon}\left(\nabla_{y} \widetilde{v}_{\varepsilon}\right)-J\left(\nabla_{y} v\right) \geq-2 \delta
$$

where $\delta>0$ is arbitrary small. The statement of the lemma then follows immediately.

7.2 Existence of a recovery sequence

Lemma 22 Let $v(x, y) \in L^{p}\left(\Omega, W_{0}^{1, p}\left(Y_{0}\right)\right)$. Then there exists a sequence $v_{\varepsilon}(x) \in$ $W_{0}^{1, p}\left(E_{0}^{\varepsilon}\right)$ such that $v_{\varepsilon} \stackrel{2}{\rightarrow} v$ and

$$
\lim _{\varepsilon \rightarrow 0} \int_{\Omega} W_{0}^{\varepsilon}\left(\varepsilon \nabla v_{\varepsilon}\right) \chi_{0}^{\varepsilon} \mathrm{d} x=\int_{\Omega} \int_{Y_{0}} Q W_{0}\left(\nabla_{y} v\right) \mathrm{d} y \mathrm{~d} x .
$$

Proof Assume first that $v \in C^{\infty}\left(\Omega \times Y_{0}\right)$. As in Lemma 21 for each $\varepsilon$ we cover the sets $\Omega$ and $Y_{0}$ by finite unions of disjoint cubes $D_{s}$ and $G_{t}$ respectively, with edge length equal to $\varepsilon$. For each $\varepsilon$ we introduce the function

$$
\widetilde{v}_{\mathcal{E}}(x, y)= \begin{cases}\frac{1}{|\varepsilon Y|} \int_{D_{s} \ni x} v(\zeta, y) d \zeta & , x \in D_{s} \subset \bar{\Omega}, \\ 0 & , x \in D_{s}: D_{s} \cap \mathbb{R}^{n} \backslash \bar{\Omega} \neq \varnothing,\end{cases}
$$

i.e. for each $x$ we take the mean of $v$ over the cube $D_{s}$ that contains $x$. The restriction of $\widetilde{v}_{\varepsilon}(x, y)$ to each $D_{s}$ is a function of $y$ only. Clearly

$$
\begin{gathered}
\left\|\widetilde{v}_{\mathcal{E}}-v\right\|_{L^{p}\left(\Omega \times Y_{0}\right)} \rightarrow 0, \\
\left\|\nabla_{y} \widetilde{v}_{\mathcal{E}}-\nabla_{y} v\right\|_{L^{p}\left(\Omega \times Y_{0}\right)} \rightarrow 0 .
\end{gathered}
$$

Notice that the function $\widetilde{v}_{\varepsilon}(x, x / \varepsilon)$ is a well-defined measurable function which belongs to ${ }^{8} W_{0}^{1, p}\left(E_{0}^{\varepsilon}\right)$. In the context of the unfolding method (see Definition 13) one has $\mathscr{T}_{\varepsilon}\left(\widetilde{v}_{\mathcal{\varepsilon}}(x, x / \varepsilon)\right)=\widetilde{v}_{\mathcal{\varepsilon}}(x, y)$. Thus we have

$$
\begin{gathered}
\int_{\Omega \times Y_{0}}\left|\widetilde{v}_{\mathcal{\varepsilon}}(x, y)\right|^{p} \mathrm{~d} y \mathrm{~d} x=\int_{E_{0}^{\varepsilon}}\left|\widetilde{v}_{\mathcal{E}}(x, x / \varepsilon)\right|^{p} \mathrm{~d} x, \\
\int_{\Omega \times Y_{0}} \widetilde{v}_{\mathcal{\varepsilon}}(x, y) g(x, y) \mathrm{d} y \mathrm{~d} x=\int_{E_{0}^{\varepsilon}} \widetilde{v}_{\mathcal{\varepsilon}}(x, x / \varepsilon) g(x, x / \varepsilon) \mathrm{d} x,
\end{gathered}
$$

for any smooth function $g$. This and (34) implies that

as $\varepsilon \rightarrow 0$.

$$
\widetilde{v}_{\varepsilon}(x, x / \varepsilon) \stackrel{2}{\rightarrow} v(x, y)
$$

Further, we approximate the function $v$ by a piecewise constant function $e_{s, t}^{\varepsilon}=$ $e_{s, t}$ given by (30) with $h=\varepsilon .{ }^{9}$ It is well know, see $e . g$. [17, Theorem 6.9], that for

\footnotetext{
${ }^{8}$ In the above construction, the cover of $\Omega$ needs to be taken to consist of the cubes $D_{s}:=$ $\varepsilon\left(Y+z_{s}\right)$, where $z_{s}$ is an integer vector.

9 Obviously, $e_{s, t}$ in (30) depends on $h$, but there we dropped it in the notation. Now, however, we would like to emphasise the dependence of the approximation $e_{s, t}^{\varepsilon}$ from $\varepsilon$.
} 
any $\delta>0$ (in particular for $\delta=\varepsilon$ ) and any $e_{s, t}^{\varepsilon}$ there exists $\varphi_{s, t}^{\varepsilon} \in W_{0}^{1, \infty}(Y)$ such that

$$
\left|Q W_{0}^{\varepsilon}\left(e_{s, t}^{\varepsilon}\right)-\int_{Y} W_{0}^{\varepsilon}\left(e_{s, t}^{\varepsilon}+\nabla \varphi_{s, t}^{\varepsilon}(z)\right) d z\right| \leq \delta=\varepsilon .
$$

Notice that due to the smoothness of $v$ the means $e_{s, t}^{\varepsilon}$ are bounded uniformly in $\varepsilon, s$ and $t$. Hence the values $Q W_{0}^{\varepsilon}\left(e_{s, t}^{\varepsilon}\right)$ are uniformly bounded as well. Therefore the functions $\varphi_{s, t}^{\varepsilon}$ are uniformly bounded in $W^{1, p}(Y)$, which follows from (36), the growth conditions on $W_{0}^{\varepsilon}$ and the Poincare inequality. A change of variables $z=y / \varepsilon$ yields

$$
\int_{Y} W_{0}^{\varepsilon}\left(e_{s, t}^{\varepsilon}+\nabla \varphi_{s, t}^{\varepsilon}(z)\right) d z=\varepsilon^{-n} \int_{\varepsilon Y} W_{0}^{\varepsilon}\left(e_{s, t}^{\varepsilon}+\varepsilon \nabla_{y} \varphi_{s, t}^{\varepsilon}(y / \varepsilon)\right) \mathrm{d} y .
$$

Let us denote by $\psi^{\varepsilon}(x, y) \in L^{p}\left(\Omega, W_{0}^{1, p}\left(Y_{0}\right)\right)$ the function whose restriction to a set $D_{s} \times G_{t}$ equals $\varepsilon \varphi_{s, t}^{\varepsilon}\left(y / \varepsilon+\xi_{t}^{\varepsilon}\right)$ if $D_{s} \times G_{t} \subset \bar{\Omega} \times \bar{Y}_{0}$ and zero otherwise, where the constant vector $\xi_{t}^{\varepsilon}$ is chosen so that $G_{t}+\xi_{t}^{\varepsilon}=\varepsilon Y$ in order to have $\varepsilon \varphi_{s, t}^{\varepsilon}(y / \varepsilon+$ $\left.\xi_{t}^{\varepsilon}\right) \in W_{0}^{1, p}\left(G_{t}\right)$. Obviously $\psi^{\varepsilon} \rightarrow 0$ in $L^{p}\left(\Omega \times Y_{0}\right)$. The fact that $\varphi_{s, t}^{\varepsilon} \in W_{0}^{1, \infty}(Y)$ and the boundedness of $\nabla_{y} \psi^{\varepsilon}$ in $L^{p}\left(\Omega \times Y_{0}\right)$ imply $\nabla_{y} \psi^{\varepsilon} \rightarrow 0$ in $L^{p}\left(\Omega \times Y_{0}\right)$. From (36) and (37) it follows that

$$
\left|\sum_{s, t} \int_{K_{s t}}\left(Q W_{0}^{\varepsilon}\left(e_{s, t}^{\varepsilon}\right)-W_{0}^{\varepsilon}\left(e_{s, t}^{\varepsilon}+\nabla_{y} \psi^{\varepsilon}\right)\right) \mathrm{d} y \mathrm{~d} x\right| \leq C \varepsilon,
$$

where the constant $C$ depends on the function $v$ and the sets $\Omega$ and $Y_{0}$. Clearly the function $\psi^{\varepsilon}(x, x / \varepsilon) \in W_{0}^{1, p}\left(E_{0}^{\varepsilon}\right)$ is measurable and satisfies equalities analogous to (35). Hence $\psi^{\varepsilon}(x, x / \varepsilon) \stackrel{2}{\rightarrow} 0$.

Consider the function $v_{\varepsilon}(x)=\widetilde{v}_{\varepsilon}(x, x / \varepsilon)+\psi^{\varepsilon}(x, x / \varepsilon)$. We claim that it satisfies the lemma. Indeed, the conditions $v_{\varepsilon}(x) \in W_{0}^{1, p}\left(E_{0}^{\varepsilon}\right)$ and $v_{\varepsilon} \stackrel{2}{\rightarrow} v$ are obviously fulfilled. In order to prove (33) we notice first that

$$
\begin{gathered}
\int_{\Omega} W_{0}^{\varepsilon}\left(\varepsilon \nabla v_{\varepsilon}\right) \chi_{0}^{\varepsilon} \mathrm{d} x-\int_{\Omega} \int_{Y_{0}} Q W_{0}\left(\nabla_{y} v\right) \mathrm{d} y \mathrm{~d} x \\
=\int_{\Omega} W_{0}^{\varepsilon}\left(\varepsilon \nabla v_{\varepsilon}\right) \chi_{0}^{\varepsilon} \mathrm{d} x-\sum_{s, t} \int_{K_{s t}} W_{0}^{\varepsilon}\left(e_{s, t}^{\varepsilon}+\nabla_{y} \psi^{\varepsilon}\right) \mathrm{d} y \mathrm{~d} x \\
+\sum_{s, t} \int_{K_{s t}}\left(W_{0}^{\varepsilon}\left(e_{s, t}^{\varepsilon}+\nabla_{y} \psi^{\varepsilon}\right)-Q W_{0}^{\varepsilon}\left(e_{s, t}^{\varepsilon}\right)\right) \mathrm{d} y \mathrm{~d} x \\
+\sum_{s, t} \int_{K_{s t}}\left(Q W_{0}^{\varepsilon}\left(e_{s, t}^{\varepsilon}\right)-Q W_{0}^{\varepsilon}\left(\nabla_{y} v\right)\right) \mathrm{d} y \mathrm{~d} x \\
+\int_{\Omega \times Y_{0}}\left(Q W_{0}^{\varepsilon}\left(\nabla_{y} v\right)-Q W_{0}\left(\nabla_{y} v\right)\right) \mathrm{d} y \mathrm{~d} x \\
=: J_{1}+J_{2}+J_{3}+J_{4} .
\end{gathered}
$$


Further, since

$$
\int_{\Omega} W_{0}^{\varepsilon}\left(\varepsilon \nabla v_{\varepsilon}\right) \chi_{0}^{\varepsilon} \mathrm{d} x=\int_{\Omega \times Y_{0}} W_{0}^{\varepsilon}\left(\nabla_{y} \widetilde{v}_{\varepsilon}+\nabla_{y} \psi^{\varepsilon}\right) \mathrm{d} y \mathrm{~d} x,
$$

and due to (6) we can estimate the first term in (39) as follows:

$$
\begin{gathered}
\left|J_{1}\right| \leq \sum_{s, t} \int_{K_{s t}}\left|W_{0}^{\varepsilon}\left(\nabla_{y} \widetilde{v}_{\varepsilon}+\nabla_{y} \psi^{\varepsilon}\right)-W_{0}^{\varepsilon}\left(e_{s, t}^{\varepsilon}+\nabla_{y} \psi^{\varepsilon}\right)\right| \mathrm{d} y \mathrm{~d} x \\
\leq \sum_{s, t} \int_{K_{s t}} \beta\left(1+\left|\nabla_{y} \widetilde{v}_{\varepsilon}+\nabla_{y} \psi^{\varepsilon}\right|^{p-1}+\left|e_{s, t}^{\varepsilon}+\nabla_{y} \psi^{\varepsilon}\right|^{p-1}\right)\left|\nabla_{y} \widetilde{v}_{\varepsilon}-e_{s, t}^{\varepsilon}\right| \mathrm{d} y \mathrm{~d} x .
\end{gathered}
$$

Hence

$$
J_{1} \rightarrow 0
$$

as $\varepsilon \rightarrow 0$, due to boundedness of $\nabla_{y} \widetilde{v}_{\varepsilon}, \nabla_{y} \psi^{\varepsilon}, e_{s, t}^{\varepsilon}$ in $L^{p}$ and the convergence properties (31), (34). The second term $J_{2}$ in (39) is already estimated, see (38). The third term $J_{3}$ can be shown to converge to zero analogously to (40). Finally, $J_{4} \rightarrow 0$ due to the pointwise convergence of $Q W_{0}^{\varepsilon}$ to $Q W_{0}$. Hence (33) holds true.

In the general case of $v(x, y) \in L^{p}\left(\Omega, W_{0}^{1, p}\left(Y_{0}\right)\right)$ we approach $v$ by a sequence of $C^{\infty}$ functions: $v_{i} \rightarrow v$ and $\nabla_{y} v_{i} \rightarrow \nabla_{y} v$ in $L^{p}\left(\Omega \times Y_{0}\right)$. For each $v_{i}$ we can apply the lemma as shown above. Then using the continuity property of the integral functional we can find the required sequence by the method of extraction of a diagonal subsequence via Proposition 17.

Combining the results of Lemma 21 and Lemma 22 we arrive at the following statement.

Theorem 23 Suppose that the family of stored-energy functions $W_{0}^{\varepsilon}, \varepsilon>0$, satisfies the conditions of Section 2. Then the two-scale $\Gamma$-limit of the functional $I_{0}^{\varepsilon}$ (see (26)) is given by

$$
\Gamma_{\text {ts }}\left(L^{p}\right)-\lim _{\varepsilon \rightarrow 0} I_{0}^{\varepsilon}(v)=I_{0}^{\mathrm{hom}}(v):=\int_{\Omega} \int_{Y_{0}} Q W_{0}\left(\nabla_{y} v\right) \mathrm{d} y \mathrm{~d} x .
$$

\section{Splitting the functional into the stiff and soft components}

In previous sections we studied two-scale $\Gamma$-limits of two integral functionals, $I_{1}^{\varepsilon}$ and $I_{0}^{\varepsilon}$, corresponding to the stiff and soft components of the elastic body. In what follows we demonstrate that the full functional $I^{\varepsilon}$ can by presented as a sum of $I_{1}^{\varepsilon}$ and $I_{0}^{\varepsilon}$ and an asymptotically small term. Consequently, the two-scale $\Gamma$-limit of $I^{\varepsilon}$ can be obtained as a sum of the two-scale $\Gamma$-limits of $I_{1}^{\varepsilon}$ and $I_{0}^{\varepsilon}$.

Consider the integral functional $I^{\varepsilon}(c f .(8))$ with the potential energy of applied forces included:

$$
I^{\varepsilon}(u)=\int_{\Omega}\left(W_{1}(\nabla u) \chi_{1}^{\varepsilon}+W_{0}^{\varepsilon}(\varepsilon \nabla u) \chi_{0}^{\varepsilon}\right) \mathrm{d} x-\int_{\Omega} f \cdot u \mathrm{~d} x .
$$


Let a sequence $u_{\varepsilon}$ be such that $I^{\varepsilon}\left(u_{\varepsilon}\right) \leq C$. Then $\left\|\nabla u_{\varepsilon} \chi_{1}^{\varepsilon}\right\|_{L^{p}(\Omega)} \leq C,\left\|\varepsilon \nabla u_{\varepsilon} \chi_{0}^{\varepsilon}\right\|_{L^{p}(\Omega)} \leq$ $C$ and hence the following representation is valid:

$$
u_{\varepsilon}=\widetilde{u}_{\varepsilon}+v_{\varepsilon}
$$

where $\left\|\nabla \widetilde{u}_{\varepsilon}\right\|_{L^{p}(\Omega)} \leq C$ and $v_{\varepsilon} \in W_{0}^{1, p}\left(E_{0}^{\varepsilon}\right)$ (see Lemma 8). We split the value of the functional $I^{\varepsilon}$ on $u_{\varepsilon}$ into the sum of two terms involving functionals $I_{1}^{\varepsilon}$ and $I_{0}^{\varepsilon}$ (cf. (24)) that correspond to the stiff and soft components and some small term (as $\varepsilon \rightarrow 0)$ :

$$
I^{\varepsilon}\left(u_{\varepsilon}\right)=\widehat{I}_{1}^{\varepsilon}\left(\widetilde{u}_{\varepsilon}\right)+\widehat{I}_{0}^{\varepsilon}\left(v_{\varepsilon}\right)+R^{\varepsilon}\left(u_{\varepsilon}\right),
$$

where

$$
\begin{gathered}
\widehat{I}_{1}^{\varepsilon}\left(\widetilde{u}_{\varepsilon}\right)=\int_{\Omega} W_{1}\left(\nabla \widetilde{u}_{\varepsilon}\right) \chi_{1}^{\varepsilon} \mathrm{d} x-\int_{\Omega} f \cdot \widetilde{u}_{\varepsilon} \mathrm{d} x, \\
\widehat{I}_{0}^{\varepsilon}\left(v_{\varepsilon}\right)=\int_{\Omega} W_{0}^{\varepsilon}\left(\varepsilon \nabla v_{\varepsilon}\right) \chi_{0}^{\varepsilon} \mathrm{d} x-\int_{\Omega} f \cdot v_{\varepsilon} \mathrm{d} x, \\
R^{\varepsilon}\left(u_{\varepsilon}\right)=\int_{\Omega}\left(W_{0}^{\varepsilon}\left(\varepsilon \nabla u_{\varepsilon}\right)-W_{0}^{\varepsilon}\left(\varepsilon \nabla v_{\varepsilon}\right)\right) \chi_{0}^{\varepsilon} \mathrm{d} x .
\end{gathered}
$$

Note that the functionals $\widehat{I}_{1}^{\varepsilon}, \widehat{I}_{0}^{\varepsilon}$ differ from $I_{1}^{\varepsilon}, I_{0}^{\varepsilon}$ only by a linear term due to the applied forces density $f$. The regularity property (6) yields

$$
\left|R^{\varepsilon}\left(u_{\varepsilon}\right)\right| \leq C \int_{\Omega}\left(1+\left|\varepsilon \nabla u_{\varepsilon}\right|^{p-1}+\left|\varepsilon \nabla v_{\varepsilon}\right|^{p-1}\right)\left|\varepsilon \nabla \widetilde{u}_{\varepsilon}\right| \chi_{0}^{\varepsilon} \mathrm{d} x .
$$

Then, via the Hölder inequality,

$$
R^{\varepsilon}\left(u_{\varepsilon}\right) \rightarrow 0 \text { as } \varepsilon \rightarrow 0,
$$

since the sequence $\nabla \widetilde{u}_{\varepsilon}$ is bounded in $L^{p}$. This immediately implies that

$$
\liminf _{\varepsilon \rightarrow 0} \inf _{u} I^{\varepsilon}(u)=\liminf _{\varepsilon \rightarrow 0} \inf _{\widetilde{u}} \widehat{I}_{1}^{\varepsilon}(\widetilde{u})+\liminf _{\varepsilon \rightarrow 0} \inf _{v} \widehat{I}_{0}^{\varepsilon}(v) .
$$

In other words one can deal with the functionals $\widehat{I}_{1}^{\varepsilon}$ and $\widehat{I}_{0}^{\varepsilon}$ separately in the context of minimising sequences. We will employ this observation in the argument of Section 10.

In addition, as was shown in Section 5 , if $u_{\varepsilon} \stackrel{2}{\rightarrow} u(x, y)$ then either the energies $I^{\varepsilon}\left(u_{\varepsilon}\right)$ converge to infinity or $u(x, y)=u_{0}(x)+v(x, y)$, where $u_{0} \in W^{1, p}(\Omega)$ and $v \in L^{p}\left(\Omega, W_{0}^{1, p}\left(Y_{0}\right)\right)$. In the latter case one has, in view of (43), (45),

$$
\begin{aligned}
\liminf _{\varepsilon \rightarrow 0} I^{\varepsilon}\left(u_{\varepsilon}\right) & \geq \liminf _{\varepsilon \rightarrow 0} \widehat{I}_{1}^{\varepsilon}\left(\widetilde{u}_{\varepsilon}\right)+\liminf _{\varepsilon \rightarrow 0} \widehat{I}_{0}^{\varepsilon}\left(v_{\varepsilon}\right) \\
& \geq I_{1}^{\text {hom }}\left(u_{0}\right)+I_{0}^{\text {hom }}(v)-\int_{\Omega} \int_{Y_{0}} f \cdot\left(u_{0}+v\right) \mathrm{d} y \mathrm{~d} x,
\end{aligned}
$$

where $I_{1}^{\text {hom }}, I_{0}^{\text {hom }}$ are defined by (25), (41).

Further, suppose that $u_{0} \in W^{1, p}(\Omega), v \in L^{p}\left(\Omega, W_{0}^{1, p}\left(Y_{0}\right)\right)$ are given. Let $v_{\varepsilon}$ be the sequence from Lemma 22 , and $\widetilde{u}_{\varepsilon}$ be such that $\widetilde{u}_{\varepsilon} \rightarrow u_{0}$ in $L^{p}(\Omega)$ as $\varepsilon \rightarrow 0$ and 
$\lim _{\varepsilon \rightarrow 0} I_{1}^{\varepsilon}\left(\widetilde{u}_{\varepsilon}\right)=I_{1}^{\mathrm{hom}}\left(u_{0}\right)$, see Theorem 19. Then, clearly, $u_{\varepsilon}:=\widetilde{u}_{\varepsilon}+v_{\varepsilon} \stackrel{2}{\rightarrow} u_{0}+v$ and (cf. (43), (45))

$$
\begin{aligned}
\lim _{\varepsilon \rightarrow 0} I^{\varepsilon}\left(u_{\varepsilon}\right) & =\lim _{\varepsilon \rightarrow 0} \widehat{I}_{1}^{\varepsilon}\left(\widetilde{u}_{\varepsilon}\right)+\lim _{\varepsilon \rightarrow 0} \widehat{I}_{0}^{\varepsilon}\left(v_{\varepsilon}\right)+\lim _{\varepsilon \rightarrow 0} R^{\varepsilon}\left(u_{\varepsilon}\right) \\
& =I_{1}^{\text {hom }}\left(u_{0}\right)+I_{0}^{\text {hom }}(v)-\int_{\Omega} \int_{Y_{0}} f \cdot\left(u_{0}+v\right) \mathrm{d} y \mathrm{~d} x .
\end{aligned}
$$

Hence, we have proved the following theorem.

Theorem 24 The two-scale $\Gamma$-limit of the functional $I^{\varepsilon}$, see (42), is given by

$$
\begin{aligned}
\Gamma_{\mathrm{ts}}\left(L^{p}\right)-\lim _{\varepsilon \rightarrow 0} I^{\varepsilon}(u) & =I^{\mathrm{hom}}(u) \\
& :=\left\{\begin{array}{c}
I_{1}^{\mathrm{hom}}\left(u_{0}\right)+I_{0}^{\mathrm{hom}}(v)-\int_{\Omega} \int_{Y_{0}} f \cdot u \mathrm{~d} y \mathrm{~d} x, \\
\text { if } u=u_{0}+v \in W^{1, p}(\Omega)+L^{p}\left(\Omega, W_{0}^{1, p}\left(Y_{0}\right)\right), \\
+\infty, \text { otherwise. }
\end{array}\right.
\end{aligned}
$$

Minimisation problem for an integral functional is not fully defined without introducing some boundary conditions. Let $\gamma$ be a measurable subset of the boundary of $\Omega$, and $\phi \in W^{1, p}(\Omega)$. Let as define a new functional $I_{\gamma, \phi}^{\varepsilon}$ satisfying boundary conditions $\phi$ on $\gamma$ as

$$
I_{\gamma, \phi}^{\varepsilon}(u):=\left\{\begin{array}{l}
I^{\varepsilon}(u) \text { if } u=\phi \text { on } \gamma \\
+\infty, \text { otherwise }
\end{array}\right.
$$

and analogously

$$
I_{\gamma, \phi}^{\mathrm{hom}}(u):=\left\{\begin{array}{l}
I^{\mathrm{hom}}(u) \text { if } u_{0}=\phi \text { on } \gamma \\
+\infty, \text { otherwise. }
\end{array}\right.
$$

Then Theorem 24 is also valid with functionals $I^{\varepsilon}$ and $I^{\text {hom }}$ replaced by their counterparts $I_{\gamma, \phi}^{\varepsilon}$ and $I_{\gamma, \phi}^{\text {hom }}$ subject to boundary conditions. This result follows from Proposition 11.7 and Remark 11.8 in [8].

While the above two-scale $\Gamma$-convergence statement fully characterises the two-scale behaviour as $\varepsilon \rightarrow 0$ of the functionals $I^{\varepsilon}$ given by (2), it is worth pointing out that at the moment there is no statement available about a priori compactness of minimising sequences for $I^{\varepsilon}$, and so the two-scale analogue of Theorem 5 does not apply. This implies that: 1) There is no automatic attainability of the infinum of the limit functional $\left.I^{\mathrm{hom}} ; 2\right)$ As far as the infima of $I^{\varepsilon}$ are concerned, only the inequality $\lim \sup _{\varepsilon \rightarrow 0} \inf I^{\varepsilon} \leq \inf I^{\text {hom }}$ is guaranteed, and further study is needed regarding the convergence of $\inf I^{\varepsilon}$ to $\inf I^{\text {hom }}$ as $\varepsilon \rightarrow 0$. In the next two sections we demonstrate that both these issues are resolved positively for the functionals (2). 


\section{Existence of minimum of the limit functional}

In the next two sections we assume that the functionals $I^{\varepsilon}$ and $I^{\text {hom }}$ are subject to boundary conditions $u=\phi, u_{0}=\phi$, on $\gamma \subset \Omega, \phi \in W^{1, p}(\Omega)$, as described at the end of Section 8 . In this section we prove that the limit functional $I^{\text {hom }}$ attains its minimum. Consider the part of the limit functional corresponding to the soft component ( $c f .(41))$

$$
\widetilde{I}_{0}^{\text {hom }}(v)=\int_{\Omega} \int_{Y_{0}} Q W_{0}\left(\nabla_{y} v\right) \mathrm{d} y \mathrm{~d} x-\int_{\Omega} \int_{Y_{0}} f \cdot v \mathrm{~d} y \mathrm{~d} x,
$$

defined on $v \in L^{p}\left(\Omega, W_{0}^{1, p}\left(Y_{0}\right)\right)$. Since $f$ depends only on $x$, we can consider $x$ as a parameter in the problem of minimisation of the functional. Thus we consider the minimisation of

$$
F_{t}(w)=\int_{Y_{0}} Q W_{0}(\nabla w) \mathrm{d} y-t \cdot \int_{Y_{0}} w \mathrm{~d} y
$$

where $w \in W_{0}^{1, p}\left(Y_{0}\right)$ and $t \in \mathbb{R}^{m}$ represents $f(x)$. It is well known that for any $t$ this functional attains its minimum. Let us denote by $\mathscr{W}(t)$ the set of all minimisers of $F_{t}$. Thus we are dealing with a set-valued function defined on $\mathbb{R}^{m}$ with values being subsets of $\left[L^{p}\left(Y_{0}\right)\right]^{m}, \mathscr{W}(t) \subset\left[L^{p}\left(Y_{0}\right)\right]^{m}$. We need to show the existence of a selection $w: \mathbb{R}^{m} \rightarrow\left[L^{p}\left(Y_{0}\right)\right]^{m}, w(t) \in \mathscr{W}(t)$, which is measurable as a function of two variables $(t, y) \in \mathbb{R}^{m} \times Y_{0}$. To this end we will employ the measurable selection theorem, but first we would like to introduce a few definitions.

Definition 25 Let $X$ be a topological space, $\mathscr{B}(X)$ be the $\sigma$-algebra of Borel sets of $X, f: \mathbb{R}^{m} \rightarrow X, F: \mathbb{R}^{m} \rightarrow\{A: A \subset X\}$. For $A \subset X$ we define its inverse image $F^{-}(A)=\{t: F(t) \cap A \neq \varnothing\}$. We say that $f$ is measurable if $f^{-1}(B)$ is measurable for $B \in \mathscr{B}(X)$. We say that $F$ is weakly measurable (as a set-valued map) if $F^{-}(B)$ is measurable for all open $B \subset X$. A map $f: \mathbb{R}^{m} \rightarrow X$ is called simple if it has finitely many values.

Let us show that $\mathscr{W}$ defined above is weakly measurable as a set-valued function. The topology in the space $L^{p}\left(Y_{0}\right)$ is determined by the underlying norm. Let $B \subset L^{p}\left(Y_{0}\right)$ be closed. Let $\left\{t_{n}\right\} \subset \mathscr{W}^{-}(B)$ be an arbitrary converging sequence, $t_{n} \rightarrow t$. For every $n$ there exists a minimiser $w_{n}$ of $F_{t_{n}}$ which belongs to $B$. The sequence $w_{n}$ is bounded in $W^{1, p}\left(Y_{0}\right)$ as it follows from the standard growth conditions for $Q W_{0}$. Then there exists a converging strongly in $L^{p}\left(Y_{0}\right)$ and weakly in $W^{1, p}\left(Y_{0}\right)$ subsequence $w_{n} \rightarrow w$ (not relabelled). It is easy to see that the sequence $F_{t_{n}} \Gamma$-converges to $F_{t}$ as $n \rightarrow \infty$, where the $\Gamma$-convergence is understood with respect to the convergence in $L^{p}\left(Y_{0}\right)$ (see Definition 3). Indeed, the lower semicontinuity type inequality follows from quasiconvexity of the integrand $Q W_{0}$ and the fact that the integral functionals with quasiconvex integrand are lower semicontinuous with respect to weak convergence in $W^{1, p}\left(Y_{0}\right)$. Existence of the recovery sequence is trivial. So by Theorem 5 the limit function $w$ is a minimiser of $F_{t}$, and, hence $w \in \mathscr{W}(t)$. On the other hand $w \in B$ since $B$ is closed set. We conclude that $t \in \mathscr{W}^{-}(B)$, that is $\mathscr{W}^{-}(B)$ is closed set. Any open set $A$ in $L^{p}\left(Y_{0}\right)$ can be presented as a countable union of closed sets $A=\cup_{i} B_{i}$. Hence its inverse 
image $\mathscr{W}^{-}(A)$ is measurable as a countable union of closed sets $\mathscr{W}^{-}\left(B_{i}\right)$. This proves that the map $\mathscr{W}$ is weakly measurable.

By the well-known measurable selection theorem (see e.g. [36, Theorem 3.1], [20]) $\mathscr{W}$ has a selection, which is measurable (in the sense of Definition 25), and we denote this selection by $w=w(t)$. By the well-known measurability criterion (see e.g. [16, Proposition E.2]) there exists a sequence $w_{n}$ of measurable simple maps such that

$$
w(t)=\lim _{n \rightarrow \infty} w_{n}(t)
$$

and

$$
\left\|w_{n}(t)\right\|_{L^{p}\left(Y_{0}\right)} \leq\|w(t)\|_{L^{p}\left(Y_{0}\right)} .
$$

The relations (47) and (48) hold for all $t$ and the limit in (47) is understood with respect to the convergence in $L^{p}\left(Y_{0}\right)$-norm. The key point here is that every simple map $w_{n}$ is Lebesgue measurable as a function of two variables $w_{n}(t, y): \mathbb{R}^{m} \times Y_{0} \rightarrow$ $\mathbb{R}^{m}$. Using the standard growth conditions and the Poincaré inequality it is easy to obtain the following estimate for $w(t)$ (as a minimiser of $F_{t}$ ):

$$
\|w(t)\|_{L^{p}\left(Y_{0}\right)}^{p} \leq\|w(t)\|_{W^{1, p}\left(Y_{0}\right)}^{p} \leq C\left(|t|^{q}+1\right) .
$$

Indeed,

$$
\begin{gathered}
\|\nabla w(t)\|_{L^{p}\left(Y_{0}\right)}^{p} \leq \int_{Y_{0}} Q W_{0}(\nabla w(t)) \mathrm{d} y=\min F_{t}+t \cdot \int_{Y_{0}} w \mathrm{~d} y \\
\leq C\left(1+|t|\|w(t)\|_{L^{p}\left(Y_{0}\right)}\right) \leq C\left(1+|t|\|\nabla w(t)\|_{L^{p}\left(Y_{0}\right)}\right),
\end{gathered}
$$

from which (49) follows.

Then from (48) and (49) it follows that $w_{n}(t, y) \in L^{p}\left(I \times Y_{0}\right)$ for any bounded set $I \subset \mathbb{R}^{m}$. Moreover, from (47) we see that $w_{n}$ is a Cauchy sequence in $L^{p}\left(I \times Y_{0}\right)$, and hence its limit in $L^{p}\left(I \times Y_{0}\right)$ coincides with $w$. Hence we conclude that the mapping $w$ is measurable as a function of two variables $w(t, y)$ and belongs to $L_{\text {loc }}^{p}\left(\mathbb{R}^{m}, W_{0}^{1, p}\left(Y_{0}\right)\right)$.

Substituting $t$ with $f(x)$ we obtain $v(x, y)=w(f(x), y), v \in L^{p}\left(\Omega, W_{0}^{1, p}\left(Y_{0}\right)\right)$, $c f$. (49). Obviously $v$ is a minimiser of $I_{0}^{\text {hom }} \cdot 10$

\section{Convergence of infima of the functionals $I^{\varepsilon}$}

Next, as was outlined at the end of Section 8, we prove that

$$
\liminf _{\varepsilon \rightarrow 0} \inf _{u} I^{\varepsilon}(u) \geq \min _{u} I^{\text {hom }}(u)
$$

where $(c f .(46))$

$$
\begin{gathered}
I^{\text {hom }}(u)=\int_{\Omega} W_{1}^{\text {hom }}\left(\nabla u_{0}\right) \mathrm{d} x+\int_{\Omega} \int_{Y_{0}} Q W_{0}\left(\nabla_{y} v\right) \mathrm{d} y \mathrm{~d} x-\int_{\Omega} \int_{Y} f \cdot u \mathrm{~d} y \mathrm{~d} x, \\
W_{1}^{\text {hom }}(e)=\liminf _{t \rightarrow \infty}\left\{\frac{1}{t^{n}} \int_{(0, t)^{n} \cap E_{1}} W_{1}(e+\nabla \varphi) \mathrm{d} x: \varphi \in W_{0}^{1, p}\left((0, t)^{n}\right)\right\}, \\
u=u_{0}(x)+v(x, y) \in W^{1, p}(\Omega)+L^{p}\left(\Omega, W_{0}^{1, p}\left(Y_{0}\right)\right) .
\end{gathered}
$$

\footnotetext{
10 Perhaps a person more familiar with measure theory might suggest a shorter proof of the existence of a measurable minimiser or even claim that this fact is "obvious".
} 
In combination with the inequality $\lim \sup _{\varepsilon \rightarrow 0} \inf I^{\varepsilon} \leq \min I^{\text {hom }}$ (which is a consequence of (46)), this will imply the equality $\lim _{\varepsilon \rightarrow 0} \inf I^{\varepsilon}=\min I^{\text {hom }}$.

Aiming to get a contradiction with (50) we assume the contrary, i.e.

$$
\liminf _{\varepsilon \rightarrow 0} \inf _{u} I^{\varepsilon}(u)<\min _{u} I^{\mathrm{hom}}(u) .
$$

Then, by the virtue of the procedure described in Section 8, there exists a sequence $u_{\varepsilon}=\widetilde{u}_{\varepsilon}+v_{\varepsilon}$ such that

$$
\begin{aligned}
\lim _{\varepsilon \rightarrow 0} I^{\varepsilon}\left(u_{\varepsilon}\right) & =\operatorname{liminfinf}_{\varepsilon \rightarrow 0} I_{u}^{\varepsilon}(u) \\
& =\liminf _{\varepsilon \rightarrow 0} \inf _{\widetilde{u}}^{\varepsilon}(\widetilde{u})+\liminf _{\varepsilon \rightarrow 0} \inf _{v} \widehat{I}_{0}^{\varepsilon}(v)<\min _{u} I^{\mathrm{hom}}(u),
\end{aligned}
$$

where $\widehat{I}_{1}^{\varepsilon}$ and $\widehat{I}_{0}^{\varepsilon}$ are as defined in (44), and $v_{\varepsilon} \stackrel{2}{\rightarrow} v(x, y), \varepsilon \nabla v_{\varepsilon} \stackrel{2}{\rightarrow} \nabla_{y} v(x, y), \widetilde{u}_{\varepsilon} \rightarrow u_{0}$ strongly in $L^{p}(\Omega)$ and weakly in $W^{1, p}(\Omega)$ for some $u_{0}$ and $v$ as in (51). Notice that, by the construction of Section $8, \widetilde{u}_{\varepsilon}$ and $v_{\varepsilon}$ are infimising sequences for $\widehat{I}_{1}^{\varepsilon}$ and $\widehat{I}_{0}^{\varepsilon}$, respectively. It is well known from the classical theory of $\Gamma$-convergence, see [8], that

$$
\begin{aligned}
\Gamma-\lim _{\varepsilon \rightarrow 0} \widehat{I}_{1}^{\varepsilon}(\psi) & =I_{1}^{\text {hom }}(\psi)-\int_{\Omega} f \cdot \psi \mathrm{d} y \mathrm{~d} x \\
& =\int_{\Omega} W_{1}^{\text {hom }}(\nabla \psi) \mathrm{d} x-\int_{\Omega} f \cdot \psi \mathrm{d} y \mathrm{~d} x, \psi \in W^{1, p}(\Omega) .
\end{aligned}
$$

Moreover,

$$
\begin{aligned}
\lim _{\varepsilon \rightarrow 0} \widehat{I}_{1}^{\varepsilon}\left(\widetilde{u}_{\varepsilon}\right)=\liminf _{\varepsilon \rightarrow 0} \widehat{I}_{1}^{\varepsilon}(u)=\min _{u}\left(I_{1}^{\mathrm{hom}}(u)-\int_{\Omega} f \cdot u \mathrm{~d} y \mathrm{~d} x\right) \\
=I_{1}^{\mathrm{hom}}\left(u_{0}\right)-\int_{\Omega} f \cdot u_{0} \mathrm{~d} y \mathrm{~d} x .
\end{aligned}
$$

Further, denote the unfolding of $v_{\varepsilon}$ by $\widehat{v}_{\varepsilon}$, i.e. $\widehat{v}_{\varepsilon}(x, y)=\mathscr{T}_{\varepsilon}\left(v_{\varepsilon}\right)(x, y)$ (see Definition 13). Since $v_{\varepsilon} \stackrel{2}{\sim} v$ and hence $\widehat{v}_{\varepsilon} \rightarrow v$ in $L^{p}(\Omega)$, one has

$$
\lim _{\varepsilon \rightarrow 0}\left\{\int_{\Omega} f \cdot v_{\varepsilon} \mathrm{d} x-\int_{\Omega} \int_{Y_{0}} f \cdot \widehat{v}_{\varepsilon} \mathrm{d} y \mathrm{~d} x\right\}=0
$$

Notice also, that $\widehat{v}_{\varepsilon} \in L^{p}\left(\Omega, W_{0}^{1, p}\left(Y_{0}\right)\right)$, and

$$
\int_{\Omega} W_{0}^{\varepsilon}\left(\nabla v_{\varepsilon}\right) \chi_{0}^{\varepsilon} \mathrm{d} x=\int_{\Omega} \int_{Y_{0}} W_{0}^{\varepsilon}\left(\nabla_{y} \widehat{v}_{\varepsilon}\right) \mathrm{d} y \mathrm{~d} x \geq \int_{\Omega} \int_{Y_{0}} Q W_{0}^{\varepsilon}\left(\nabla_{y} \widehat{v}_{\varepsilon}\right) \mathrm{d} y \mathrm{~d} x .
$$

Clearly, from (46), (52), (53), (54) and (55) it follows that for sufficiently small $\varepsilon$ one has

$$
I^{\mathrm{hom}}\left(u_{0}+\widehat{v}_{\varepsilon}\right)<\min _{u} I^{\mathrm{hom}},
$$

which is a contradiction. Hence (50) is valid. This, together with Theorem 24, implies the following statement 
Theorem 26 For the functionals $I^{\varepsilon}$ defined by (2) under the conditions of Section 2, one has

$$
\liminf _{\varepsilon \rightarrow 0} I^{\varepsilon}(u)=\min _{u} I^{\mathrm{hom}}(u),
$$

where $I^{\text {hom }}$ is defined by (46). For any minimiser $u$ of $I^{\text {hom }}$ there exists a sequence $u_{\varepsilon}$ such that $u_{\varepsilon} \stackrel{2}{\rightarrow} u$ and

$$
\lim _{\varepsilon \rightarrow 0} I^{\varepsilon}\left(u_{\varepsilon}\right)=I^{\mathrm{hom}}(u)
$$

\section{Concluding remarks}

Here we offer a discussion of our results hoping to place them better in the context of the existing literature, and suggest possible avenues for further development.

It may be worth highlighting first the synergy of several known analytical methods within our approach, notably the two-scale convergence, periodic unfolding method and the concept of $\Gamma$-convergence. There is a number of previous works where these methods have been used in treating what we would like to refer to as "classical" models, whereby the macroscopic, homogenised, solution depends only on the slow variable. Importantly however, these approaches have a multi-scale character in that a priori they may assume a two-scale nature of the leading order term in the behaviour of the solutions as $\varepsilon \rightarrow 0$. For example, the two-scale convergence, either on it own or in combination with the procedure of unfolding, leads in the limit to functions that in general depend both on the slow and fast variable, and it is only by virtue of the standard $W^{1, p_{-}}$ compactness that the single-scale structure of the limit is inferred a posteriori. The $\Gamma$-convergence method can then be viewed as a complementary tool that allows to deal with problems that may not have a weak formulation, in particular problems with non-convex energies. From this perspective our work fills an existing gap in the mathematical theory of homogenisation by demonstrating how the method of $\Gamma$-convergence can be used to analyse problems where the two-scale structure of the solution persists "to the very limit" as $\varepsilon \rightarrow 0$. A basic example of such a setting is that of high-contrast composites, which fail to satisfy the property of uniform coercivity as $\varepsilon \rightarrow 0$, and hence do not possess the usual $W^{1, p}$-compactness property. Prior to our work such problems had only been studied within settings that are amenable to direct treatment via the definition of the two-scale convergence (confusingly enough, this would correspond to what is referred to as the indirect approach in the calculus of variations). In view of the fundamental importance of those physical settings that are underpinned by some kind of energy minimisation principle, as e.g. the setting of static nonlinear elasticity, one is led to asking whether "non-classical" non-convex problems, in particular those that exhibit high-contrast in the constitutive response at different material points, can be analysed by combining the two-scale and variational approaches. Thus one arrives at the concept of "two-scale $\Gamma$-convergence", which we define in Section 5 and which exploits two-scale, rather than one-scale, compactness properties of variational integrals.

It is perhaps appropriate to note here that as an abstract concept, the two-scale $\Gamma$-convergence is not new: some elements of it have appeared, e.g., in the works 
[34], [35], [21]. The rationale leading up to the related theory becomes transparent when one notices that the usual $L^{p}$-norm convergence and the strong twoscale convergence are equivalent under the unfolding transformation, and hence the "two-scale" $\Gamma$-convergence can be defined using the standard $\Gamma$-convergence in two-variable $L^{p}$ spaces. While this approach yields the desired effective material description and is a convenient tool of studying solutions to those problems where lack of compactness is not an issue, in the non-classical settings a more direct approach may be preferable where one deals with sequences of functions in the original function space $\left(L^{p}(\Omega)\right.$ in our case) rather than those that are the result of unfolding. Convergence statements for oscillating sequences of a given twoscale functional are then formulated in terms of the sequences themselves and not in terms their "unfolded" counterparts whose limits would require "folding back" to the original variables.

The technique presented in this paper is aimed at treating high-contrast functionals of the "double-porosity" type, in that the underlying periodic composite is made of two types of materials, "stiff" and "soft", whose individual constitutive relations exhibit a high degree (of order $\varepsilon$ ) of contrast between each other and which occupy certain domains within the unit cell. While the individual storedenergy function for each of these two contrasting material types may vary "moderately" over the unit cell leaving the mathematical statements within our work essentially unchanged, large (i.e. tending to infinity as $\varepsilon \rightarrow 0$ ) variations within each type are not covered by our theory. This restriction is manifested by the fact that the stored-energy functions $W^{\varepsilon}=W^{\varepsilon}(y, e)$ for the composite family in question are postulated to have the form $W^{\varepsilon}(y, e)=W_{0}(y, e) \chi_{0}(y)+W_{1}(y, \varepsilon e) \chi_{1}(y)$, which corresponds to the strict separation between the "stiff" and "soft" materials. Another limitation of our results is that at present they only apply to materials that are either isotropic or at worst weakly anisotropic at each point in space, i.e. the contrast in their constitutive response in different spatial directions does not get large as $\varepsilon \rightarrow 0$. Extending the two-scale $\Gamma$-convergence to highly anisotropic (or "partially degenerate") materials (see the works [12], [30] in the linearised setting) would be an important step forward, in our opinion, both from the mathematical and the applied perspective, in view of their potential for applications as nonlinear metamaterials. The key difficulty in doings so within the present framework is posed by the inability to consider the stiff and soft parts of the related integral functional separately from each other when treating such partially degenerate situations.

From the point of view of the mathematical theory of nonlinear elasticity, there is one significant drawback of the our technique as described, namely the fact that the growth condition (4) is incompatible with the requirement that $W^{\varepsilon}(y, e) \rightarrow \infty$ as $\operatorname{det}(e) \rightarrow 0+$. The associated technical difficulties, however, are present even in the "moderate contrast" setting of the standard mathematical elasticity, see [2]. In particular, even the issues of the existence of minimisers and the validity of the weak Euler-Lagrange equation are known to be open for non-polyconvex functionals of this kind even in the absence of periodic microstructure. Hence, in our work we feel justified to restrict ourselves to the case of the usual global growth condition (4), awaiting further progress on these problems elsewhere. It should perhaps be mentioned, however, that the theory we propose allows for minimising sequences that are "discontinuous at the microscale": an example of these is 
obtained by taking e.g. a cavitating minimiser of the "soft" part of the limiting functional. This feature seems to be inherently out of reach by the "classical" theories.

One general analytical issue that is raised by our theory is whether one can strengthen Lemma 21 (Section 7.1) by proving the inequality $I^{\text {hom }}(u) \leq \liminf _{\varepsilon \rightarrow 0} I^{\varepsilon}\left(u_{\varepsilon}\right)$ with respect to the weak two-scale convergence. A simple example showing that the answer to this question is negative, is via a "checkerboard" sequence $u_{\varepsilon}$ constructed by taking a functional $I^{\mathrm{hom}}$ whose soft component admits two different global minimisers $\psi_{1}$ and $\psi_{2}$, setting $u_{\varepsilon}=\psi_{i}(x / \varepsilon)$ within the soft part of each periodicity cell of size $\varepsilon$ so that the values of the index $i$ in any two neighbouring cells are different, and extending $u_{\varepsilon}$ to zero elsewhere. The possible non-compactness of a minimising sequence for $I^{\varepsilon}$ is illustrated using the same example: clearly the sequence $u_{\varepsilon}$ weakly two-scale converges to $\chi_{0}(y)\left(\psi_{1}(y)+\psi_{2}(y)\right) / 2$ but it fails to converge in the strong two-scale sense. Clearly, in the case when the stored-energy function $W_{0}$ is convex, this kind of counterexample is not possible, and for such functionals we expect a two-scale analogue the Mosco convergence to hold (see e.g. [23])

Our technique calls for an extension in various directions. In addition to the case of partially degenerate composites already mentioned, one could consider classes of stored-energy functions with different growth exponents for the "stiff" and "soft" components, as well as combinations of high-contrast periodic with "thin" structures, where the volume fraction of the stiff component vanishes in the limit as $\varepsilon \rightarrow 0$. The technique of " $\Gamma$-developments", which can be thought of as an analogue of the method of asymptotic expansions in the nonlinear setting (see [10] and references therein), should also be amenable to an extension using the two-scale $\Gamma$-convergence approach presented here, to the contexts where the two-scale behaviour persists to the limit as $\varepsilon \rightarrow 0$. We plan to address these issues elsewhere.

Acknowledgements The support of the EPSRC under the grant EP/F03797X/1 is gratefully acknowledged by both authors.

\section{References}

1. Allaire, G.: Homogenization and two-scale convergence. SIAM J. Math. Anal. 20(6), 1482$1518(1992)$

2. Ball, J.M.: Some open problems in elasticity. In: Geometry, Mechanics, and Dynamics, Springer-Verlag, New York, 3-59 (2002)

3. Bakhvalov N.S., Panasenko, G.: Homogenisation: Averaging Processes in Periodic Media, Mathematics and Its Applications (Soviet Series), 36, Kluwer Academic Publishers, Dordrecht, Boston, London, 1989

4. Bensoussan, A., Lions, J.-L., Papanicolaou, G.: Asymptotic Analysis of periodic Structures North Holland, Ansterdam, 1978

5. Bouchitté, G., Bellieud, M.: Homogenization of a soft elastic material reinforced by fibers. Asymptot. Anal. 32(2), 153-183 (2002)

6. Bouchitté, D., Felbacq, D.: Homogenisation near resonances and artificial magnetism from dielectrics. C. R. Math. Acad. Sci. Paris 339(5), 337-382 (2004)

7. Braides, A.: Homogenization of some almost periodic coercive functionals. Rend. Accad Naz. Sci. XL 103, 313-322 (1985)

8. Braides, A., Defranceschi, A.: Homogenization of Multiple Integrals. Oxford Lecture Series in Mathematics and Its Applications 12, New York: Oxford University Press, 1998 
9. Braides, A., Piat, V.C., Piatnitski, A.: A variational approach to double-porosity problems. Asymptot. Anal. 39(3-4), 281-308 (2004)

10. Braides, A., Truskinovsky, L.: Asymptotic expansions by $\Gamma$-convergence. Cont. Mech. Thermodyn., 20(1), 21-62 (2008)

11. Briane, M., Casado-Daz, J., Murat. F.: The div-curl lemma "trente ans aprés": an extension and an application to the $G$-convergence of unbounded monotone operators. J. Math. Pures Appl. 91(5), 476-494 (2009)

12. Cherednichenko, K.D., Smyshlyaev, V.P., Zhikov, V.V.: Non-local homogenised limits for composite media with highly anisotropic periodic fibres. Proc. Royal Soc. Edinurgh, Sect. A Math., 136(1) 87-114 (2006)

13. Cioranescu, D., Damlamian, A., Griso, G.: Periodic unfolding and homogenization. $C$. $R$. Acad. Sci. Paris, Sr. I Math. 335, 99104 (2002)

14. Cioranescu, D., Damlamian, A., Griso, G.: The Periodic Unfolding Method in Homogenization. SIAM Journal on Mathematical Analysis 40(4), 1585-1620 (2008)

15. Cioranescu, D., Donato, P.: An Introduction to Homogenization. Oxford University Press, Oxford, 1999.

16. Cohn, D.L.: Measure theory. Birkhäuser, 1980

17. Dacorogna, B.: Direct Methods in the Calculus of Variations. Springer-Verlag, New-York, 1989

18. De Giorgi, E., Spagnolo, S.: Sulla convergenza degli integrali dell'energia per operatori ellittici del secondo ordine. (Italian) Boll. Un. Mat. Ital. 8(4), 391-411 (1973)

19. Jikov, V.V., Kozlov, S.M., Oleinik, O.A.: Homogenisation of Differential Operators and Integral Functionals. Springer, 1994

20. Kuratowski K., Ryll-Nardzewski C.: A general theorem on selectors. Bull. Polish Acad. Sci. 13, 397-403 (1965)

21. Mielke, A., Timofte, A.: Two-scale homogenization for evolutionary variational inequalities via the energetic formulation. SIAM J. Math. Anal., 39(2), 642-668 (2007)

22. Milton, G.W.: Composite materials with Poisson's ratios close to -1. J. Mech. Phys. Solids 40, 1105-1137 (1992)

23. Mosco, U.: Convergence of convex sets and of solutions of variational inequalities. Advances in Mathematics, 3, 510-585 (1969)

24. Müller, S.: Homogenization of nonconvex integral functionals and cellular elastic materials. Arch. Rational Mech. Anal. 99, 189-212 (1987)

25. Murat, F.: Compacité par compensation. Ann. Scuola Norm. Sup. Pisa Cl. Sci. (4) 5, 489 507 (1978)

26. Nguetseng, G.: A general convergence result for a functional relate to the theory of homogenization. SIAM J. Math. Anal. 20, 608-623 (1989)

27. Nicorovici, N.A., McPhedran, R.C., Milton, G.W.: Optical and dielectric properties of partially resonant composites. Phys. Rev. B 49, 8479-8482 (1994)

28. Pendry, J.B.: Negative refraction makes a perfect lens. Phys. Rev. Lett. 85, 3966-3969 (2000)

29. Rudin, W.: Functional Analysis. McGraw-Hill Science, 1991

30. Smyshlyaev, V.P.: Propagation and localization of elastic waves in highly anisotropic periodic composites via two-scale homogenization. Mechanics of Materials, 41(4), 434-447 (2009)

31. Tartar, L.: Problèmes de côntrole descoefficients dans des équations aux dérivées partielles. In: Lecture Notes in Economics and Mathematical Systems 107, 420-426 (1974)

32. Tartar, L.: Compensated compactness and applications to partial differential equations. Nonlinear Analysis and Mechanics, Heriot-Watt Symposium, IV (R. J. Knops, ed.), Pitman Res. Notes Math. Ser., Longman Sci. Tech., Harlow, 136-192 (1979)

33. Veselago, V.G.: The electrodynamics of substances with simultaneously negative values of $\varepsilon$ and $\mu$. Soviet Physics Uspekhi 10, 509-514 (1968)

34. Visintin, A.: Towards a two-scale calculus. ESAIM, Control Optim. Calc. Var., 12, 371-397 (2006)

35. Visintin, A.: Two-scale convergence of some integral functionals. Calc. Var. Partial Differ. Equ., 29(2), 239-265 (2007)

36. Wagner, D.H.: Survey of measurable selection theorems: An update. In "Lecture Notes in Mathematics No. 794," Springer-Verlag, New York/Berlin, 176-219 (1980)

37. Zhikov, V.V.: On the extension of the method of two-scale convergence and its applications. Sb. Math. 191(7), 973-1014 (2000) 
38. Zhikov, V.V.: Solvability of Generalised Navier-Stokes Equations. Doklady Mathematics 78(3), 1-5 (2000)

39. Zhikov, V.V.: A note on Sobolev spaces. J. Math. Sci. 129(1), 3593-3595 (2005)

40. Zhikov, V.V.: Solvability of the three-dimensional thermistor problem. Proceedings of the Steklov Institute of Mathematics, 261, 1-14 (2008)

41. Zhikov, V.V., Rychago M.E., Shul'ga, S.B.: Homogenisation of monotone operators by the method of two-scale convergence. J. Math. Sci. 127(5), 2159-2173 (2005) 